\title{
Antiangiogenic Effect of Alkaloids
}

\author{
Masoud Alasvand, ${ }^{1}$ Vahideh Assadollahi, ${ }^{2}$ Roberto Ambra, ${ }^{3}$ Ehsan Hedayati, ${ }^{4}$ \\ Wesam Kooti $\left(\mathbb{D},{ }^{5} \text { and Ilaria Peluso }{ }^{3}\right)^{3}$ \\ ${ }^{1}$ Department of Medical Physiology and Pharmacology, Faculty of Medicine, Kurdistan University of Medical Sciences, \\ Sanandaj, Iran \\ ${ }^{2}$ Cellular and Molecular Research Center, Research Institute for Health Development, Kurdistan University of Medical Sciences, \\ Sanandaj, Iran \\ ${ }^{3}$ Council for Agricultural Research and Economics, Research Centre for Food and Nutrition (CREA-AN), Via Ardeatina 546, \\ 00178 Rome, Italy \\ ${ }^{4}$ Student Research Committee, Ahvaz Jundishapur University of Medical Sciences, Ahvaz, Iran \\ ${ }^{5}$ Cellular and Molecular Research Center, Sabzevar University of Medical Sciences, Sabzevar, Iran
}

Correspondence should be addressed to Wesam Kooti; wesamkooti@gmail.com

Masoud Alasvand and Vahideh Assadollahi contributed equally to this work.

Received 30 November 2018; Revised 6 March 2019; Accepted 17 March 2019; Published 21 April 2019

Guest Editor: Simona Bungau

Copyright (c) 2019 Masoud Alasvand et al. This is an open access article distributed under the Creative Commons Attribution License, which permits unrestricted use, distribution, and reproduction in any medium, provided the original work is properly cited.

\begin{abstract}
Alkaloids are among the natural phytochemicals contained in functional foods and nutraceuticals and have been suggested for the prevention and/or management of oxidative stress and inflammation-mediated diseases. In this review, we aimed to describe the effects of alkaloids in angiogenesis, the process playing a crucial role in tumor growth and invasion, whereby new vessels form. Antiangiogenic compounds including herbal ingredients, nonherbal alkaloids, and microRNAs can be used for the control and treatment of cancers. Several lines of evidence indicate that alkaloid-rich plants have several interesting features that effectively inhibit angiogenesis. In this review, we present valuable data on commonly used alkaloid substances as potential angiogenic inhibitors. Different herbal and nonherbal ingredients, introduced as antiangiogenesis agents, and their role in angiogenesisdependent diseases are reviewed. Studies indicate that angiogenesis suppression is exerted through several mechanisms; however, further investigations are required to elucidate their precise molecular and cellular mechanisms, as well as potential side effects.
\end{abstract}

\section{Introduction}

Alkaloids are among the natural phytochemicals contained in functional foods and nutraceuticals [1] and have been suggested for the prevention and/or management of oxidative stress and inflammation-mediated diseases $[1,2]$. In this review, we aimed to describe the effect of alkaloids on angiogenic vessel formation from a previous existing capillary, a process that is implicated in many physiological conditions such as wound healing and menstrual cycle and pathological conditions such as tumor growth or retinopathy [3-5].
The angiogenic process is a cascade of events resulting in new lumen-containing vessels, through the dissolution of the vascular basal membrane, migration of endothelial cells from the parent vessel toward developing blood vessels, and widespread cellular proliferation, which are activated by several proangiogenic factors. When blood flow is initiated, physiological processes such as embryonic development, wound healing, and immune reactions are then allowed to start and develop [6, 7]. On the other hand, the creation of new blood vessels increases the supply of nutrients, oxygen, and growth factors to normal and tumor cells. If tumor cells can 
induce angiogenesis, subsequent tumor expansion and transition from a benign state to a malignant one is started [8]. Endothelial cells can migrate in order to initiate or progress to angiogenesis, thanks to a wide range of regulators and signaling molecules such as basic fibroblast growth factor (bFGF), epidermal growth factor (EGF), vascular endothelial growth factor (VEGF), hepatocyte growth factor (HGF), and transforming growth factor (TGF). Thus, these angiogenic peptides play a critical role in the creation of new blood vessels [9-11]. In both physiological and pathological conditions, HIF-1 is a critical mediator of hypoxic response and $\mathrm{O}_{2}$ homeostasis as well as an essential angiogenic regulator. Hypoxia leads to HIF- $1 \alpha$ stabilization, a subunit of the heterodimeric transcription factor HIF-1, and increased VEGF production $[12,13]$. Oxygen demand can be increased by excessive cell proliferation during embryonic development and/or tumor growth [14].

Moreover, VEGF is the main factor that initiates angiogenesis. Similar to some proangiogenic genes, VEGF also has hypoxia response element (HRES) as the binding site of HIF-1 within the promoters. In hypoxic conditions, HIF-1 directly stimulates VEGF and its receptors, inducing angiogenesis $[15,16]$. The abovementioned mechanisms in the formation of new blood vessels demonstrate that VEGF directly links hypoxia with angiogenesis initiation. The VEGF family of growth factors exerts its effects by interacting with receptor tyrosine kinases (RTKs) named vascular endothelial growth factor receptors (VEGFRs). Activation of this signaling pathway leads to endothelial cell (EC) proliferation, extracellular matrix degradation, EC migration, and then new blood vessel formation $[17,18]$. Understanding the tumor angiogenesis signaling pathways is an important treatment goal of malignancy and cancer therapy. However, because of the similarities between tumor and physiologic angiogenesis signaling pathways, insufficient efficacy and resistance may be challenges we face during such cancer therapy [19].

The results of different studies show that several molecular pathways such as VEGFRs, Ephrin-Eph receptors, and the Delta-like ligand and neurogenic locus notch homolog protein (Delta-Notch) system are involved in angiogenesis $[7,20]$.

\section{Angiogenesis Signaling Pathways}

VEGF belongs to the platelet-derived growth factor (PDGF) family; moreover, the VEGF family has five members in mammals, including VEGF-A, VEGF-B, VEGF-C, VEGF$\mathrm{D}$, and PlGF (placental growth factor). All of them stimulate cellular responses and are characterized by the presence of eight conserved cysteine residues forming the typical cysteine-knot structure [21]. VEGFRs are tyrosine kinase receptors (TKRs) with a cytoplasmic domain and tyrosine kinase activity, containing 3 to 4 members. VEGF-A, the most important member of this family, was obtained from tumor cells for the first time. VEGF-A regulates angiogenesis and vascular permeability via activation of VEGFR-1 and VEGFR-2. The binding of VEGF-A to its receptor represents the most important signal for angiogenesis. On the other hand, VEGF-C/VEGF-D and their receptor, VEGFR-3, are involved in lymphangiogenesis [22, 23]. Several VEGF family ligands and receptors, especially VEGF-A, are regulated by HIF; therefore, angiogenesis is intensified during healthy tissue growth and also in cancerous conditions [24]. Following the binding of VEGF-A to VEGFR2, the RAS/RAF (rapidly accelerated fibrosarcoma)/ERK (extracellular signalregulated kinases)/MAPK (mitogen-activated protein kinases) pathway is activated potentially via sphingosine kinase (SPK) activated by protein kinase $\mathrm{C}$ (PKC). RAS target genes can now be enabled, inducing proliferation [25]. Recent studies consider the role of the transcription factor nuclear factorkappa beta $(\mathrm{NF}-\kappa \mathrm{B})$, which interacts with RAS, in tumor angiogenesis; therefore, it can be an antiangiogenic therapeutic target. It has been demonstrated that oncogenic RAS mediates the activation of the NF- $\kappa$ B pathway and subsequent upregulation of NF- $\kappa \mathrm{B}$ target genes, in particular the proangiogenic IL-8 cytokine, leading to lung tumorigenesis [26].

Other RAS-independent molecular pathways, such as the phospholipase C gamma- (PLC $\gamma$-) protein kinase C- (PKC-) MAPK pathway, are also implicated in VEGFR2 signaling [22]. Binding of VEGFR2 to VEGF-A and phosphorylation of several tyrosine residues in the cytoplasmic domain of this receptor can induce the activation of phospholipase $\mathrm{C}$ gamma (PLC $\gamma)$. On the one hand, PLC $\gamma$ releases diacylglycerol (DAG) which recruits PKC to the cell membrane and promotes cell proliferation; on the other hand, PLC $\gamma$ mediated $\mathrm{Ca}^{2+}$ mobilization leads to increased vascular permeability, promoting leakage of plasma proteins into the extravascular space, resulting in a fibrin gel formation marking the proper temporary place for the placement of the new blood vessel, and supporting its growth [23, 27, 28].

Generally, the phosphorylation of different tyrosines in the cytoplasmic domain of VEGFR2 and the subsequent recruitment of signal transducers promote migration and cell mobility and regulate cell attachment, all of which are needed for angiogenesis [29]. As already mentioned, different molecular pathways are involved in pathophysiological angiogenesis compared to the physiological one [30]. The phosphatidylinositol 3-kinase (PI3K)/AKT/mammalian target of rapamycin (mTOR) pathway is activated in many human cancers. The activation of the PI3K/AKT pathway in cancer cells can increase VEGF production. This may be done through HIF-1-dependent mechanisms, or it may occur in an HIF-1-independent manner. In tumor cells, PI3K/AKT activation can occur because of RAS mutation which is responsible for tumor cell feeding [12]. Other signaling pathways, such as JAK-STAT, can promote the migration of endothelial cells and subsequent tumor angiogenesis [31].

On the other hand, VEGFR1 is a negative regulator of angiogenesis. VEGFR1 is a decoy receptor, and the binding of VEGF-A to it may prevent the activation of VEGFR2. Also, Delta-like ligand 4 (DII4), a transmembrane ligand of notch family receptors, is a negative regulator of angiogenesis. More precisely, when VEGF binds to VEGFR2, it causes DII4 upregulation which in turn interacts with NOTCH1 receptors on adjacent endothelial cells, resulting in VEGFR2 downregulation in those cells. Finally, the activation of Notch signaling leads to a reduced number of tip cells and less dense 
vascular network formation via the prevention of tubular sprouting in angiogenesis $[22,32]$.

The involvement of coagulation factors in tumor-induced and normal angiogenesis has been reported in several studies $[33,34]$, supporting their role in tumor progression.

\section{Angiogenesis Inhibitors and Cancer Treatment}

Angiogenesis is the creation of new capillaries from preexisting vessels and circulating endothelial precursors [35]. It can be activated in physiological and pathological conditions, for example, in response to hypoxia, cancer, diabetic retinopathy, and inflammatory, infectious, and immune disorders [36]. Proangiogenic growth factors commonly switch on a series of surface receptors in a series of paracrine and autocrine circles with the VEGF-A signaling representing the critical rate-limiting step, physiologically and pathologically. VEGF-A is the main VEGF that binds to VEGFR2 to mediate vascular permeability, endothelial proliferation, migration, and survival [37].

Angiogenesis also plays a critical role in the growth of cancer for the reason that cancer tissues need a blood supply. Tumors can stimulate normal cells to generate angiogenesis signaling molecules. Angiogenesis inhibitors act through several ways. Some of them are monoclonal antibodies that recognize and bind to VEGF. When VEGF is attached to these drugs, it is unable to activate the VEGF receptor [38].

Other inhibitors bind to the VEGF receptor on the surface of endothelial cells or to other proteins in the downstream signaling pathways and block their activities. Some of the inhibitors are immunomodulatory drug agents that stimulate or suppress the immune system. One of the ideas of antiangiogenic therapy is through the destruction tumor vessels [39].

Since angiogenesis is the main requisite for cancer growth and invasion, its inhibition is considered the mainstay in cancer treatment strategies. The study of angiogenesis led to the identification of numerous proangiogenic factors as well as angiogenic suppressor proteins. By the identification of these proteins, a wide spectrum of angiogenesis inhibitors were introduced in cancer treatment procedures. Several antiangiogenic molecules currently in use employ anti-VEGF-A antibodies [40, 41] and tyrosine kinase inhibitors [42-45]. However, serious adverse effects have been reported for such molecules [46]. On the other hand, herbal drugs, most commonly alkaloid-based plants, are among the more promising angiogenic inhibitors to be used for the treatment of different types of cancers like prostate cancer cells, breast cancer cells, melanoma cells, and cervical cancer cells [47-51].

\section{Role of MicroRNAs in Angiogenesis Regulation}

The identification of proteins involved in angiogenic processes led to the identification of microRNAs (miRNAs) as potent inhibitors of angiogenesis and cancer. MiRNAs are single-stranded short noncoding RNA molecules (about 2125 nucleotides) that modulate cellular target genes at the posttranscriptional level. These RNA molecules have a role in almost all biological processes and their potential role in most pathogenesis has been observed. Cancers and thrombotic and bleeding disorders, as well as heart disease, are among those disorders in which the potential role of miRNAs has been investigated. Defects in miRNA synthesis led to a wide spectrum of early disorders including impaired T-cell formation, cardiomyopathy, disrupted blood circulation, and impaired angiogenesis [52-55]. One of the most important consequences of the impaired biogenesis of miRNAs is early embryonic lethality due to defective vessel formation and maintenance [56-58]. These observations encouraged studies to evaluate the role of different miRNAs in angiogenic processes, and several miRNAs were identified with a potential role in the regulation of different aspects of angiogenesis.

It has been reported that some of the miRNAs, such as miR-126, miR-221/222, miR-17-92 cluster, miR-93, let-7f, and miR-214, modulate the response of the vascular endothelium to angiogenic stimuli and can be proangiogenic or antiangiogenic [59-62].

MiR-221 and miR-222 are two relatively well-known miRNAs with an important effect on protooncogene c-Kit (CD117) as a key role in the survival of hematopoietic stem cells. Further studies on endothelial cells revealed that these two miRNAs have antiangiogenic activity $[63,64]$. Overexpression of miR-221 and miR-222 in endothelial cells led to increased endothelial nitric oxide synthase, which in turn resulted in the suppression of endothelial survival and angiogenesis. MiR-34a is another miRNA with potential antiangiogenic activity, as it was shown to induce endothelial progenitor cell (EPC) senescence and dysfunction. EPCs have a crucial role in angiogenesis and are important for many physiological processes, even pathological ones, such as the growth of tumor cells $[65,66]$. MiR-34a suppresses silent information regulator 1 (Sirt1) resulting in senescence induction in EPCs [67]. Mir-126 is another miRNA with direct and indirect effects on angiogenesis that is mainly found in tissues with high vascular components, such as the lungs and the heart [68-70]. MiR-126 regulates many endothelial cell functions and processes mainly including cell migration and cell survival. MiR-93 is involved with the stimulation of angiogenesis and the promotion of tumor growth, and its inhibition is suggested as a tool for the suppression of angiogenesis and tumor growth [71, 72].

Endothelial cells express VEGF receptor genes. miRNAs can modulate angiogenesis by regulating VEGF receptor expression in endothelial cells, whereupon they influence the migration and invasion of cancer cells. In addition, recent studies showed that Dicer and Drosha, two enzymes that control the processing of miRNAs, play key roles in the angiogenic process. Not only do miRNAs modulate mRNA translation level, but mRNA also regulates the function of miRNAs. Thus, researchers can target the molecular pathways, taking part in the development of diseases, by determining miRNAs which are important in the angiogenic process $[57,59,73,74]$.

The number of known miRNAs with a direct or indirect role in angiogenesis is on the rise, and a summary is shown in Table 1. 
TABLE 1: Effect of different microRNAs on the angiogenic process.

\begin{tabular}{|c|c|c|c|c|}
\hline MiRNA & Target gene & Role & Function & Reference \\
\hline MiR-34a & $\begin{array}{l}\text { Silent information } \\
\text { regulator } 1 \text { (Sirt1) }\end{array}$ & $\begin{array}{l}\text { MiR-34a has been found to target silent } \\
\text { information regulator } 1 \text { (Sirt1), leading to } \\
\text { cell cycle arrest or apoptosis }\end{array}$ & Antiangiogenesis & {$[67]$} \\
\hline MiR-107 & HIF- $1 \beta$ & $\begin{array}{l}\text { MiR-107 decreases hypoxia signaling by suppressing } \\
\text { expression HIF-1 } \beta\end{array}$ & Antiangiogenesis & {$[75]$} \\
\hline MiR-132 & p120RasGAP & $\begin{array}{c}\text { MiR-132 acts as an angiogenic switch by targeting } \\
\text { p120RasGAP in the endothelium and thereby } \\
\text { inducing neovascularization }\end{array}$ & Angiogenesis & {$[76]$} \\
\hline MiR-424 & Cullin 2 (CUL2) & $\begin{array}{l}\text { MiR-424 targeted Cullin } 2 \text { (CUL2), a scaffolding } \\
\text { protein critical to the assembly of the ubiquitin } \\
\text { ligase system, thereby stabilizing HIF- } \alpha \text { isoforms }\end{array}$ & Angiogenesis & [77] \\
\hline MiR-93 & Integrin- $\beta 8$ & $\begin{array}{l}\text { MiR-93 promotes angiogenesis by suppressing } \\
\text { integrin- } \beta 8 \text { expression }\end{array}$ & Angiogenesis & {$[71]$} \\
\hline MiR-29b & MMP-2 & $\begin{array}{l}\text { MiR-29b exerted its antiangiogenesis function, } \\
\text { at least partly, by suppressing MMP-2 expression } \\
\text { in tumor cells }\end{array}$ & Antiangiogenesis & {$[78]$} \\
\hline MiR-519c & HIF- $1 \alpha$ & $\begin{array}{l}\text { Overexpression of miR-519c resulted in a } \\
\text { significant decrease of HIF- } 1 \alpha \text { protein levels } \\
\text { and reduced the tube formation of human } \\
\text { umbilical vein endothelial cells }\end{array}$ & Antiangiogenesis & [79] \\
\hline MiR-210 & VEGF and VEGFR & $\begin{array}{l}\text { Overexpression of miR- } 210 \text { enhances VEGF and } \\
\text { VEGFR2 expression and promotes angiogenesis }\end{array}$ & Angiogenesis & {$[80]$} \\
\hline MiR-155 & Von Hippel-Lindau (VHL) & $\begin{array}{c}\text { MiR-155 has a pivotal role in tumor angiogenesis } \\
\text { by downregulation of VHL }\end{array}$ & Angiogenesis & {$[81]$} \\
\hline MiR-195 & VEGF, VAV2, CDC42 & $\begin{array}{l}\text { MiR-195 directly inhibited the expression of the } \\
\text { proangiogenic factor VEGF and the prometastatic } \\
\text { factors VAV2 and CDC42 }\end{array}$ & Antiangiogenesis & {$[82]$} \\
\hline MiR-145 & $H I F-2 \alpha$ & $\begin{array}{l}\text { MiR-145 suppresses } H I F-2 \alpha \text { expression, thus } \\
\text { inhibiting the angiogenesis }\end{array}$ & Antiangiogenesis & {$[83]$} \\
\hline MiR-26a & $\begin{array}{l}\text { HGF-hepatocyte growth } \\
\text { factor receptor (cMet) }\end{array}$ & $\begin{array}{l}\text { MiR-26a exerted its antiangiogenesis function, } \\
\text { at least in part, by inhibiting HGF-hepatocyte growth } \\
\text { factor (cMet) and its downstream signaling pathway }\end{array}$ & Antiangiogenesis & {$[84]$} \\
\hline MiR-214 & $\begin{array}{l}\text { Hepatoma-derived growth } \\
\text { factor (HDGF) }\end{array}$ & $\begin{array}{c}\text { Downregulation of miR-214 contributes to the unusual } \\
\text { hypervascularity of HCC via activation of the HDGF } \\
\text { paracrine pathway for tumor angiogenesis }\end{array}$ & Antiangiogenesis & {$[85]$} \\
\hline MiRNA-24 & eNOS & $\begin{array}{l}\text { Inhibition of microRNA-24 improves reparative } \\
\text { angiogenesis in myocardial infarction }\end{array}$ & Antiangiogenesis & {$[86]$} \\
\hline MiR-29a & $\begin{array}{l}\text { Phosphatase and tensin } \\
\text { homolog (PTEN) }\end{array}$ & $\begin{array}{l}\text { TGF- } \beta \text {-regulated miRNA in promoting angiogenesis } \\
\text { by targeting PTEN to stimulate AKT activity }\end{array}$ & Angiogenesis & {$[87]$} \\
\hline MiR-27b & $\begin{array}{l}\text { Vascular endothelial } \\
\text { growth factor C (VEGFC) }\end{array}$ & $\begin{array}{l}\text { MiRNA-27b targets vascular endothelial growth } \\
\text { factor } \mathrm{C} \text { to inhibit angiogenesis in colorectal cancer }\end{array}$ & Antiangiogenesis & {$[88]$} \\
\hline MiR-503 & FGF2 and VEGF-A & $\begin{array}{c}\text { Demonstrate the antiangiogenesis role of miR-503 } \\
\text { in tumorigenesis and provide a novel mechanism } \\
\text { for hypoxia-induced FGF2 and VEGF-A through } \\
\text { HIF1 } \alpha \text {-mediated inhibition of miR-503 }\end{array}$ & Antiangiogenesis & [89] \\
\hline MiR-143 & $\begin{array}{l}\text { Insulin-like growth factor-I } \\
\text { receptor (IGF-IR) }\end{array}$ & $\begin{array}{l}\text { Overexpression of miR-143 inhibited cell } \\
\text { proliferation, migration, tumor growth, and } \\
\text { angiogenesis and increased chemosensitivity to } \\
\text { oxaliplatin treatment in an IGF-IR-dependent manner }\end{array}$ & Antiangiogenesis & {$[90]$} \\
\hline MiR-382 & $\begin{array}{l}\text { Phosphatase and tensin } \\
\text { homolog (PTEN) }\end{array}$ & $\begin{array}{l}\text { MiR-382 induced by hypoxia promotes angiogenesis } \\
\text { and acts as an angiogenic oncogene by repressing PTEN }\end{array}$ & Angiogenesis & {$[91]$} \\
\hline MiR-210 & $\begin{array}{l}\text { Vascular endothelial growth factor } \\
\text { (VEGF) }\end{array}$ & $\begin{array}{l}\text { MiR-210 is a key factor at the microRNA level } \\
\text { in promoting angiogenesis and neurogenesis, which } \\
\text { was associated with local increased vascular } \\
\text { endothelial growth factor (VEGF) levels }\end{array}$ & Angiogenesis & {$[92]$} \\
\hline
\end{tabular}


TABle 1: Continued.

\begin{tabular}{|c|c|c|c|c|}
\hline MiRNA & Target gene & Role & Function & Reference \\
\hline MiR-542-3p & Angiopoietin-2 (Angpt2) & $\begin{array}{c}\text { MiR-542-3p inhibited translation of Angpt } 2 \text { mRNA } \\
\text { by binding to its } 3^{\prime} \text { UTR, and the addition of } \\
\text { miR-542-3p to cultured endothelial cells attenuated } \\
\text { angiogenesis }\end{array}$ & Antiangiogenesis & {$[93]$} \\
\hline MiR-214 & Quaking & $\begin{array}{l}\text { MiR-214 directly targets Quaking, a protein } \\
\text { critical for vascular development. Quaking } \\
\text { knockdown reduced proangiogenic growth } \\
\text { factor expression and inhibited endothelial cell } \\
\text { sprouting similar to miR-214 overexpression }\end{array}$ & Antiangiogenesis & {$[94]$} \\
\hline MiR-20a & p300 & $\begin{array}{l}\text { P300 drives an angiogenic transcription program } \\
\text { during hypertrophy that is fine-tuned in part through } \\
\text { direct repression of p300 by miR-20a }\end{array}$ & Antiangiogenesis & {$[95]$} \\
\hline MiR-15a & FGF2 and VEGF & $\begin{array}{l}\text { MiR-15a negatively regulates angiogenesis in vivo } \\
\text { and in vitro by suppression of FGF2 and VEGF }\end{array}$ & Antiangiogenesis & {$[96]$} \\
\hline
\end{tabular}

TABLE 2: Classification of different alkaloids based on amino acid precursors and their origins.

\begin{tabular}{|c|c|c|c|}
\hline Amino acid precursors & Tyrosine & Tryptophan & Lysine \\
\hline \multirow{11}{*}{ Alkaloid origins } & Berberine & Brucine & Matrine \\
\hline & Hydrastis canadensis; Coptis chinensis & Strychnos nux-vomica L. & Sophora alopecuroides \\
\hline & Noscapine & Evodiamine & Capsaicin \\
\hline & Papaver somniferum L. & Evodia rutaecarpa & Capsicum annum L. \\
\hline & Sanquinarine & Homoharringtonine & Halofuginone \\
\hline & Sanquinaria canadenis L. & Cephalotoxus & Dichroa febrifugus \\
\hline & Sinomenine & & Pterogynidine \\
\hline & Sinomenium acutum & & Pterogyne nitens Tul \\
\hline & Taspine & & \\
\hline & Tetrandarine & & \\
\hline & Stephania tetrandra S. & & \\
\hline
\end{tabular}

\section{Plant-Based Alkaloids}

Nowadays, a wide variety of plants that are rich in alkaloids have been discovered and these compounds have a significant impact on angiogenesis-dependent diseases. Alkaloids have an antiangiogenic activity and act through several mechanisms inhibiting angiogenesis. There are many alkaloids, but this review takes into account only the most important ones. By considering the hypothetical amino acid precursors and origins, different plant-based alkaloids in use are sorted into different groups. Table 2 summarizes the different alkaloids.

Table 3 summarizes studies that evaluated the effect of different alkaloids on angiogenesis in different types of disorders.

Almost all alkaloids show an antiproliferative and cytotoxic activity against cancer cell lines derived from several different histological origins (esophagus, stomach, colon, liver, lung, breast, bone, and brain), and this activity depends also on the activation of the expression of apoptotic genes [161]. On the other hand, in vitro studies showed that antiangiogenic effects rely on some shared ability to downregulate, in the same cancer cells, VEGF, TNF- $\alpha$, and HIF- $1 \alpha$ messengers and/or proteins levels (with mechanisms ranging from low expression and higher degradation), blocking the angiogenic cascade in endothelial cells, as shown for berberine [102, 106], noscapine [97], brucine [140], evodiamine [146], homoharringtonine [149], matrine [162], and tetrandrine [136]. For instance, studies have shown that the cascade is blocked by matrine and tetrandrine at the levels of STAT3 signaling ([163] and [164], respectively), while evodiamine inhibits $\beta$-catenin [165]. Similar results were reported for sanguinarine, capsaicin, taspine, harmine, and pterogynidine, for whose deeper dissection of the molecular pathways demonstrated that antiangiogenic activities specifically involve Akt phosphorylation [138, 166, 167], CDK expression [168], and NF- $\kappa \mathrm{B}$ translocation [160, 169] and that the effect is dose-dependent $(10-300 \mathrm{nM})$ [166]. Other studies indicate that some alkaloids (sinomenine, brucine, and halofuginone) are able to directly regulate in vitro, at $\mu \mathrm{M}$ concentrations, and in vivo in transplanted mice, the expression of angiogenic factors ([123, 140] and [170], respectively). In particular, sinomenine and halofuginone should be able to induce Smad protein depletion $([157,171])$.

Even if Ning et al. found that tetrandrine modulated, in human hypertrophic scar fibroblasts, the expression of miRNAs predicted to be related to wound healing [172], a process that is tightly related with angiogenesis [173], very little data 
TABLE 3: Effects of different alkaloids on angiogenesis.

\begin{tabular}{|c|c|c|c|c|c|c|c|}
\hline Name of alkaloid & $N$ & Molecules & & $\begin{array}{l}\text { Biological } \\
\text { results }\end{array}$ & Sample type & Year & Author \\
\hline \multirow{8}{*}{ Noscapine } & 1 & HIF-1 & Decrease & $\begin{array}{c}\text { Inhibit } \\
\text { angiogenesis by } \\
\text { downregulating } \\
\text { VEFG }\end{array}$ & U87MG and T98G cell lines & 2006 & Newcomb et al. [97] \\
\hline & 2 & VEGF & Decrease & $\begin{array}{c}\text { Inhibit } \\
\text { angiogenesis }\end{array}$ & Human T98 cell lines & 2008 & Newcomb et al. [98] \\
\hline & 3 & VEGF & Decrease & $\begin{array}{c}\text { Inhibit } \\
\text { angiogenesis }\end{array}$ & $\begin{array}{l}\text { Murine GL261 glioma } \\
\text { cell lines }\end{array}$ & 2008 & Newcomb et al. [98] \\
\hline & 4 & $\begin{array}{l}\text { MMP-9 } \\
\text { VEGF }\end{array}$ & Decrease & $\begin{array}{c}\text { Inhibit } \\
\text { angiogenesis }\end{array}$ & $\begin{array}{l}\text { KBM-5, HL-60, Jurkat, } \\
\text { HuT-78, U266, RPMI- 8226, } \\
\text { H1299, A293, DU145, } \\
\text { and SCC4 cell lines }\end{array}$ & 2010 & Sung et al. [99] \\
\hline & 5 & VEGF & Decrease & $\begin{array}{c}\text { Inhibit } \\
\text { angiogenesis }\end{array}$ & The A549 or H460 cell & 2010 & Chougule et al. [100] \\
\hline & 6 & VEGF & Decrease & $\begin{array}{c}\text { Inhibit } \\
\text { angiogenesis }\end{array}$ & $\begin{array}{l}\text { Xenografted with H460 } \\
\text { tumors (mice) }\end{array}$ & 2010 & Chougule et al. [100] \\
\hline & 7 & HIF & Decrease & $\begin{array}{c}\text { Inhibit } \\
\text { angiogenesis }\end{array}$ & Ovarian cancer cells & 2011 & Su et al. [101] \\
\hline & 8 & $\begin{array}{l}\text { NFKB } \\
\text { VEGF }\end{array}$ & Decrease & $\begin{array}{c}\text { Inhibit } \\
\text { angiogenesis }\end{array}$ & $\begin{array}{l}\text { The human breast } \\
\text { cancer cell } \\
\text { lines MDA-MB-231 } \\
\text { and MDA-MB-468 }\end{array}$ & 2011 & Chougule et al. [100] \\
\hline \multirow{14}{*}{ Berberine } & 9 & HIF-1 & Decrease & $\begin{array}{c}\text { Inhibit } \\
\text { angiogenesis by } \\
\text { downregulating } \\
\text { VEGF }\end{array}$ & $\begin{array}{l}\text { Gastric adenocarcinoma } \\
\text { cell line SC-M1 }\end{array}$ & 2004 & Lin et al. [102] \\
\hline & 10 & VEGF & Decrease & $\begin{array}{l}\text { Inhibit } \\
\text { angiogenesis }\end{array}$ & HeLa cell & 2008 & Lin et al. [103] \\
\hline & 11 & $\begin{array}{l}\text { VEGF } \\
\text { MMP-2 }\end{array}$ & Decrease & $\begin{array}{c}\text { Inhibit } \\
\text { angiogenesis }\end{array}$ & $\begin{array}{l}\text { Human umbilical vein } \\
\text { endothelial cells }\end{array}$ & 2009 & Gao et al. [104] \\
\hline & 12 & $\begin{array}{l}\text { Mmp-2/9 } \\
\text { UPA }\end{array}$ & Decrease & $\begin{array}{c}\text { Inhibit } \\
\text { angiogenesis }\end{array}$ & Squamous cancer cell & 2009 & Ho et al. [105] \\
\hline & 13 & VEGF & Decrease & $\begin{array}{l}\text { Inhibit } \\
\text { angiogenesis }\end{array}$ & $\begin{array}{l}\text { Hepatocellular } \\
\text { carcinoma }\end{array}$ & 2010 & C. Cheung et al. \\
\hline & 14 & VEGF & Decrease & $\begin{array}{c}\text { Inhibit } \\
\text { angiogenesis }\end{array}$ & Hep G2 cell line & 2011 & Jie et al. [106] \\
\hline & 15 & $\begin{array}{l}\text { HIF-1 } \\
\text { VEGF }\end{array}$ & Decrease & $\begin{array}{c}\text { Inhibit } \\
\text { angiogenesis }\end{array}$ & $\begin{array}{l}\text { B16F-10 melanoma cells } \\
\text { and C57BL/6 mice }\end{array}$ & 2012 & Hamsa and Kuttan [107] \\
\hline & 16 & VEGF & Decrease & $\begin{array}{c}\text { Inhibit } \\
\text { angiogenesis }\end{array}$ & $\begin{array}{c}\text { Xenografted } \\
\text { hepatocellular } \\
\text { carcinoma (mice) }\end{array}$ & 2012 & Ruhua [108] \\
\hline & 17 & VEGF & Decrease & $\begin{array}{c}\text { Inhibit } \\
\text { angiogenesis }\end{array}$ & $\begin{array}{l}\text { Hepatocellular } \\
\text { carcinoma }\end{array}$ & 2012 & Wang et al. \\
\hline & 18 & VEGF & Decrease & $\begin{array}{c}\text { Inhibit } \\
\text { angiogenesis }\end{array}$ & Breast cancer & 2013 & Kim et al. [109] \\
\hline & 19 & VEGF & Decrease & $\begin{array}{c}\text { Inhibit } \\
\text { angiogenesis }\end{array}$ & HepG2 cells & 2013 & Wang and Ke [110] \\
\hline & 20 & VEGF & Decrease & $\begin{array}{c}\text { Inhibit } \\
\text { angiogenesis }\end{array}$ & $\begin{array}{l}\text { Human non-small-cell } \\
\text { lung cancer }\end{array}$ & 2013 & Fu et al. [111] \\
\hline & 21 & $\begin{array}{l}\text { Mmp-2 } \\
\text { UPA }\end{array}$ & Decrease & $\begin{array}{c}\text { Inhibit } \\
\text { angiogenesis }\end{array}$ & Cervical cancer & 2014 & Chu et al.[112] \\
\hline & 22 & VEGF & Decrease & $\begin{array}{c}\text { Inhibit } \\
\text { angiogenesis }\end{array}$ & $\begin{array}{l}\text { Hepatocellular } \\
\text { carcinoma }\end{array}$ & 2015 & Tsang et al. [113] \\
\hline
\end{tabular}


TABle 3: Continued.

\begin{tabular}{|c|c|c|c|c|c|c|c|}
\hline Name of alkaloid & $N$ & Molecules & & $\begin{array}{l}\text { Biological } \\
\text { results }\end{array}$ & Sample type & Year & Author \\
\hline \multirow{10}{*}{ Sanguinarine } & 23 & VEGF & Decrease & $\begin{array}{c}\text { Inhibit } \\
\text { angiogenesis }\end{array}$ & Swine granulosa cell & 2006 & Bianco et al. [114] \\
\hline & 24 & VEGF & Decrease & $\begin{array}{l}\text { Inhibit } \\
\text { angiogenesis }\end{array}$ & Endothelial cell line & 2007 & Basini et al. [115] \\
\hline & 25 & VEGF & Decrease & $\begin{array}{c}\text { Inhibit } \\
\text { angiogenesis }\end{array}$ & Swine granulosa cells & 2007 & Basini et al. [116] \\
\hline & 26 & VEGF & Decrease & $\begin{array}{c}\text { Inhibit } \\
\text { angiogenesis }\end{array}$ & $\begin{array}{c}\text { Porcine aortic } \\
\text { endothelial cell line }\end{array}$ & 2007 & Basini et al. [117] \\
\hline & 27 & VEGF & Decrease & $\begin{array}{c}\text { Inhibit } \\
\text { angiogenesis }\end{array}$ & Swine ovarian follicles & 2008 & Basini et al. [116] \\
\hline & 28 & $?$ & $?$ & $\begin{array}{l}\text { Inhibit } \\
\text { angiogenesis }\end{array}$ & B16 melanoma 4 A5 cells & 2009 & De Stefano et al. [118] \\
\hline & 29 & VEGF & Decrease & $\begin{array}{l}\text { Inhibit } \\
\text { angiogenesis }\end{array}$ & $\begin{array}{l}\text { Human A549 lung } \\
\text { cancer cells }\end{array}$ & 2013 & Xu et al. [119] \\
\hline & 30 & VEGF & Decrease & $\begin{array}{c}\text { Inhibit } \\
\text { angiogenesis }\end{array}$ & $\begin{array}{l}\text { MCF-7 human } \\
\text { mammary } \\
\text { adenocarcinoma cells }\end{array}$ & 2013 & Dong et al. [120] \\
\hline & 31 & VEGF & Decrease & $\begin{array}{l}\text { Inhibit } \\
\text { angiogenesis }\end{array}$ & S180 sarcoma in mice & 2014 & Du et al. [121] \\
\hline & 32 & HIF-1 & Decrease & $\begin{array}{c}\text { Inhibit } \\
\text { angiogenesis }\end{array}$ & Pancreatic cancer & 2015 & Singh et al. [122] \\
\hline \multirow[b]{2}{*}{ Sinomenine } & 33 & VEGF & Decrease & $\begin{array}{c}\text { Inhibit } \\
\text { angiogenesis }\end{array}$ & $\begin{array}{c}\text { Human synovial } \\
\text { sarcoma cells (Hs701.T) }\end{array}$ & 2006 & Li et al. [123] \\
\hline & 34 & VEGF & Decrease & $\begin{array}{c}\text { Inhibit } \\
\text { angiogenesis }\end{array}$ & $\begin{array}{c}\text { Umbilical vein } \\
\text { endothelial cells } \\
\text { (HUVEC) and U2OS cells }\end{array}$ & 2016 & Xie et al. [124] \\
\hline \multirow{8}{*}{ Taspine } & 35 & VEGF & Decrease & $\begin{array}{c}\text { Inhibit } \\
\text { angiogenesis }\end{array}$ & $\begin{array}{c}\text { Chicken chorioallantoic } \\
\text { membrane (CAM) } \\
\text { neovascularization } \\
\text { model and CAM } \\
\text { transplantation } \\
\text { tumor model }\end{array}$ & 2008 & Zhang et al. [125] \\
\hline & 36 & VEGF & Decrease & $\begin{array}{c}\text { Inhibit } \\
\text { angiogenesis }\end{array}$ & $\begin{array}{l}\text { Human umbilical vein } \\
\text { endothelial cells }\end{array}$ & 2008 & Zhao et al. [126] \\
\hline & 37 & VEGF & Decrease & $\begin{array}{c}\text { Inhibit } \\
\text { angiogenesis }\end{array}$ & $\begin{array}{l}\text { Human umbilical vein } \\
\text { endothelial cells }\end{array}$ & 2010 & Zhang et al. [127] \\
\hline & 38 & VEGF & Decrease & $\begin{array}{l}\text { Inhibit } \\
\text { angiogenesis }\end{array}$ & Caco-2 cell lines & 2011 & Zhang et al. [128] \\
\hline & 39 & VEGF & Decrease & $\begin{array}{l}\text { Inhibit } \\
\text { angiogenesis }\end{array}$ & $\begin{array}{l}\text { Human liver cancer } \\
\text { SMMC7721 }\end{array}$ & 2011 & Zhang et al. [129] \\
\hline & 40 & VEGF & Decrease & $\begin{array}{c}\text { Inhibit } \\
\text { angiogenesis }\end{array}$ & $\begin{array}{l}\text { Chicken chorioallantoic } \\
\text { membrane (CAM) and } \\
\text { mouse colon tissue }\end{array}$ & 2012 & Zhang et al. [130] \\
\hline & 41 & VEGF & Decrease & $\begin{array}{c}\text { Inhibit } \\
\text { angiogenesis }\end{array}$ & $\begin{array}{c}\text { Human cell lines of } \\
\text { SMMC-7721, A549, } \\
\text { MCF-7, Lovo, and } \\
\text { ECV304 }\end{array}$ & 2012 & Zheng et al. [131] \\
\hline & 42 & VEGF & Decrease & $\begin{array}{c}\text { Inhibit } \\
\text { angiogenesis }\end{array}$ & A549 cells lung cancer & 2012 & Lu et al. [132] \\
\hline Tetrandrine & 43 & PDGF & Decrease & $\begin{array}{c}\text { Inhibit } \\
\text { angiogenesis }\end{array}$ & $\begin{array}{l}\text { Adjuvant-induced } \\
\text { chronic inflammation } \\
\text { model of mouse }\end{array}$ & 1998 & Kobayashi et al. [133] \\
\hline
\end{tabular}


TABle 3: Continued.

\begin{tabular}{|c|c|c|c|c|c|c|c|}
\hline Name of alkaloid & $N$ & Molecules & & $\begin{array}{l}\text { Biological } \\
\text { results }\end{array}$ & Sample type & Year & Author \\
\hline & 44 & VEGF & Decrease & $\begin{array}{c}\text { Inhibit } \\
\text { angiogenesis }\end{array}$ & RT-2 glioma RT & 2009 & Chen et al. [134] \\
\hline & 45 & $\begin{array}{l}\text { HIF-1 } \\
\text { VEGF }\end{array}$ & Decrease & $\begin{array}{l}\text { Inhibit } \\
\text { angiogenesis }\end{array}$ & $\begin{array}{c}\text { A rat model corneal } \\
\text { neovascularization } \\
(\mathrm{CNV})\end{array}$ & 2011 & Sun et al. [135] \\
\hline & 46 & $\begin{array}{l}\text { HIF-1 } \\
\text { VEGF }\end{array}$ & Decrease & $\begin{array}{c}\text { Inhibit } \\
\text { angiogenesis }\end{array}$ & $4 \mathrm{~T} 1$ tumor bearing mice & 2013 & Gao et al. [136] \\
\hline & 47 & - & - & $\begin{array}{l}\text { Inhibit } \\
\text { angiogenesis }\end{array}$ & $\begin{array}{l}\text { Human umbilical vein } \\
\text { endothelial cells } \\
\text { (HUVECs) and the } \\
\text { human colon cancer } \\
\text { cell line Lovo }\end{array}$ & 2013 & Qian et al. [137] \\
\hline & 48 & - & - & $\begin{array}{c}\text { Inhibit } \\
\text { angiogenesis }\end{array}$ & $\begin{array}{l}\text { Liver cancer xenograft } \\
\text { model in nude mice }\end{array}$ & 2015 & Xiao et al. [138] \\
\hline \multirow{7}{*}{ Brucine } & 49 & $\begin{array}{l}\text { VEGF } \\
\text { TGF- } \beta\end{array}$ & Decrease & $\begin{array}{l}\text { Inhibit } \\
\text { angiogenesis }\end{array}$ & $\begin{array}{l}\text { Murine cannulated } \\
\text { sponge implant } \\
\text { angiogenesis model }\end{array}$ & 2011 & Agrawal et al. [139] \\
\hline & 50 & VEGF & Decrease & $\begin{array}{l}\text { Inhibit } \\
\text { angiogenesis }\end{array}$ & $\begin{array}{l}\text { Ehrlich ascites tumor and } \\
\text { human cancer cell line }\end{array}$ & 2011 & Agrawal et al. [140] \\
\hline & 51 & VEGF & Decrease & $\begin{array}{c}\text { Inhibit } \\
\text { angiogenesis }\end{array}$ & $\begin{array}{c}\text { Nude mouse model of } \\
\text { bone metastasis due to } \\
\text { breast cancer }\end{array}$ & 2012 & Li et al. [141] \\
\hline & 52 & HIF-1 & Decrease & $\begin{array}{l}\text { Migration and } \\
\text { metastasis and } \\
\text { angiogenesis }\end{array}$ & $\begin{array}{l}\text { Hepatocellular } \\
\text { carcinoma }\end{array}$ & 2013 & Shu et al. [142] \\
\hline & 53 & VEGF & Decrease & Angiogenesis & Colon cancer cells & 2013 & Luo et al. [143] \\
\hline & 54 & VEGF & Decrease & $\begin{array}{c}\text { Inhibit } \\
\text { angiogenesis }\end{array}$ & Lovo cell & 2013 & Zheng et al. [144] \\
\hline & 55 & VEGF & Decrease & $\begin{array}{c}\text { Inhibit } \\
\text { angiogenesis }\end{array}$ & $\begin{array}{l}\mathrm{R} \text { breast cancer cell line } \\
\text { MDA-MB- } 231\end{array}$ & 2013 & $\begin{array}{c}\text { Kechun and Zjauma } \\
{[145]} \\
\end{array}$ \\
\hline \multirow{3}{*}{ Evodiamine } & 56 & VEGF & Decrease & $\begin{array}{l}\text { Inhibit } \\
\text { angiogenesis }\end{array}$ & $\begin{array}{c}\text { Human lung } \\
\text { adenocarcinoma } \\
\text { cell (CL1 cells) }\end{array}$ & 2006 & Shyu et al. [146] \\
\hline & 57 & VEGF & Decrease & $\begin{array}{l}\text { Inhibit } \\
\text { angiogenesis }\end{array}$ & Breast cancer cell & 2008 & Wang et al. [147] \\
\hline & 58 & HIF & Decrease & $\begin{array}{c}\text { Inhibit } \\
\text { angiogenesis }\end{array}$ & Human colon cancer cell & 2015 & Huang et al. [148] \\
\hline \multirow{4}{*}{ Homoharringtonine } & 59 & VEGF & Decrease & $\begin{array}{c}\text { Inhibit } \\
\text { angiogenesis }\end{array}$ & $\begin{array}{c}\text { Human umbilical vein } \\
\text { endothelial cell line } \\
\text { (ECV304) }\end{array}$ & 2004 & Ye and Lin [149] \\
\hline & 60 & VEGF & Decrease & $\begin{array}{c}\text { Inhibit } \\
\text { angiogenesis }\end{array}$ & $\begin{array}{l}\text { Leukemic cell line (K562) } \\
\text { in vitro }\end{array}$ & 2004 & Ye and Lin [149] \\
\hline & 61 & VEGF & Decrease & $\begin{array}{c}\text { Inhibit } \\
\text { angiogenesis }\end{array}$ & Myeloid leukemia cells & 2005 & Fei and Zhang [150] \\
\hline & 62 & HIF & Decrease & $\begin{array}{c}\text { Inhibit } \\
\text { angiogenesis }\end{array}$ & K562 cell & 2008 & Li et al. [151] \\
\hline Matrine & 63 & VEGF & Decrease & $\begin{array}{c}\text { Inhibit } \\
\text { angiogenesis }\end{array}$ & Breast cancer cell & 2009 & Yu et al. [152] \\
\hline \multirow{2}{*}{ Halofuginone } & 64 & $\begin{array}{l}\text { VEGF } \\
\text { MMP }\end{array}$ & Decrease & $\begin{array}{c}\text { Inhibit } \\
\text { angiogenesis }\end{array}$ & $\mathrm{BALB} / \mathrm{c} v / v$ (nude) mice & 2003 & Gross et al. [153] \\
\hline & 65 & - & - & $\begin{array}{c}\text { Inhibit } \\
\text { angiogenesis }\end{array}$ & $\begin{array}{l}\text { Metastatic rat brain } \\
\text { tumor model }\end{array}$ & 2004 & Abramovitch et al. [154] \\
\hline
\end{tabular}


TABle 3: Continued.

\begin{tabular}{|c|c|c|c|c|c|c|c|}
\hline Name of alkaloid & $N$ & Molecules & & $\begin{array}{l}\text { Biological } \\
\text { results }\end{array}$ & Sample type & Year & Author \\
\hline & 66 & TGF & Decrease & $\begin{array}{c}\text { Inhibit } \\
\text { angiogenesis }\end{array}$ & $\begin{array}{l}\text { Polyoma middle } \mathrm{T} \\
\text { antigen mouse }\end{array}$ & 2005 & Yee et al. [155] \\
\hline & 67 & $\begin{array}{l}\text { TGF- } \beta-1, \text { bFGF, } \\
\text { COL1A1, MMP-2, } \\
\text { MMP-9, VEGF, } \\
\text { and PDGF }\end{array}$ & Decrease & $\begin{array}{l}\text { Inhibit } \\
\text { angiogenesis }\end{array}$ & Xenografted (rat) & 2012 & Jordan and Zeplin [156] \\
\hline & 68 & $\begin{array}{c}\text { VEGF } \\
\text { MMP } \\
\text { TGF- } \beta \\
\end{array}$ & Decrease & $\begin{array}{c}\text { Inhibit } \\
\text { angiogenesis }\end{array}$ & $\begin{array}{l}\text { Acute promyelocytic } \\
\text { leukemia mouse model }\end{array}$ & 2015 & Assis et al. [157] \\
\hline \multirow{2}{*}{ Capsaicine } & 69 & VEGF & Decrease & $\begin{array}{c}\text { Inhibit } \\
\text { angiogenesis }\end{array}$ & $\begin{array}{l}\text { Endothelial cells in the } \\
\text { rat aorta }\end{array}$ & 2008 & Pyun et al. [158] \\
\hline & 70 & VEGF & Decrease & $\begin{array}{c}\text { Inhibit } \\
\text { angiogenesis }\end{array}$ & $\begin{array}{c}\text { Non-small-cell lung } \\
\text { cancer }\end{array}$ & 2014 & Chakraborty et al. [159] \\
\hline Pterogynidine & 71 & - & - & $\begin{array}{c}\text { Inhibit } \\
\text { angiogenesis }\end{array}$ & $\begin{array}{l}\text { Human umbilical vein } \\
\text { endothelial cells } \\
\text { (HUVEC) }\end{array}$ & 2009 & Lopes et al. [160] \\
\hline
\end{tabular}

is available at the moment on the role of miRNAs in alkaloidassociated angiogenic processes. One exception is for berberine that was shown to promote angiogenesis directly through miR-29b upregulation, upon induction of ischemia in mice [174]. The rest of the available data is restricted to experiments analyzing alkaloids as effectors of miR induction in different animal or cell models; for example, a recent study analyzed miRNA induction by berberine in porcine embryos [175]. Similarly, a berberine-containing commercially prepared traditional Chinese medicine Huang-Lian-Jiee-DwuTang [176] was shown to induce VEGF and miR-126 expression in mesenchymal stem cell exosomes [177]. Interestingly, a recent paper suggests that berberine, together with evodiamine, is able to affect not only the expression of some miRs in colorectal cancer cells (in particular miR-29a) but also the expression of DNA methyltransferases that in turn regulate miRs' activities, providing evidence for epigenetic activities of alkaloids during carcinogenesis [178]. In other searches, the pulmonary arterial hypertension- (PAH-) inducing plant pyrrolizidine alkaloid monocrotaline was shown to affect miR-21 and let-7a expression in the lung of monocrotalineinjected rats [179]. The role of miR-21 in PAH was later confirmed in humans [180], but no data is available on miR-21's role on monocrotaline effects. Nonetheless, downregulation of miR-21, followed by the dephosphorylation of its target Akt, was reported also by the growth-inhibiting alkaloid matrine, on breast cancer cells [181]. Similar involvement of miR-21 by alkaloids was observed on human thyroid cancer cells [182], while other miRs were involved in other cells, i.e., miR-19b in melanoma [183], miR-106b in human acute T-cell lymphoblastic leukemia [184], and miR-126 in nonsmall-cell lung cancer [185].

Kaymaz et al. found that Capsaicin strongly reduced miR-520a expression in chronic myeloid leukemia cells [186]. As the regulation is associated with the inhibition of cell proliferation, the authors hypothesized that alkaloid's apoptotic properties could rely directly on miR-520a5 p/STAT3 interaction [186]. Similarly, data on rat hepatic stellate T6 cells suggest that the ability of sanguinarine to induce apoptosis through BCL2 downregulation could rely on the activation of miR-15a/16-1 expression [187]. On the other hand, homoharringtonine antileukemic effects were causatively related, by ectopic expression, to the activation of miR-370 and its target FoxM1, a major regulator in cell proliferation and apoptosis [188]. Other transfection experiments with miR mimics demonstrated unequivocally that inhibitory activities on invasion and metastasis by sinomenine on breast cancer cells depend on miR-324-5p and involve downstream NF- $\kappa \mathrm{B}$ [189].

\section{Conclusion}

Based on data reviewed here and their ability to act as regulatory agents, alkaloids from natural sources are promising molecules with an enormous potential for therapeutic intervention of many disorders. Compounds from natural sources are believed to be good candidates for the prevention of angiogenic disorders and also as complements for chemotherapy or radiotherapy, in combination with other anticancer therapies [50, 190-192]. Berberine, noscapine, sanguinarine, and taspine among others are the most interesting and promising angiogenesis inhibitors. Further investigations are still required to elucidate their precise molecular and cellular mechanisms, as well as any potential side effects.

\section{Conflicts of Interest}

There are no conflicts of interest.

\section{Authors' Contributions}

Masoud Alasvand and Vahideh Assadollahi contributed equally to this manuscript. 


\section{References}

[1] S. A. Adefegha, "Functional foods and nutraceuticals as dietary intervention in chronic diseases; novel perspectives for health promotion and disease prevention," Journal of Dietary Supplements, vol. 15, no. 6, pp. 977-1009, 2018.

[2] M. A. Islam, F. Alam, M. Solayman, M. I. Khalil, M. A. Kamal, and S. H. Gan, "Dietary phytochemicals: natural swords combating inflammation and oxidation-mediated degenerative diseases," Oxidative Medicine and Cellular Longevity, vol. 2016, Article ID 5137431, 25 pages, 2016.

[3] N. S. Greaves, K. J. Ashcroft, M. Baguneid, and A. Bayat, "Current understanding of molecular and cellular mechanisms in fibroplasia and angiogenesis during acute wound healing," Journal of Dermatological Science, vol. 72, no. 3, pp. 206-217, 2013.

[4] L. Østergaard, A. Tietze, T. Nielsen et al., "The relationship between tumor blood flow, angiogenesis, tumor hypoxia, and aerobic glycolysis," Cancer Research, vol. 73, no. 18, pp. 5618-5624, 2013.

[5] M. Alasvand, S. H. Javanmard, B. Rashidi, and M. Khazaei, "Myocardial capillary density after neuropeptide Y antagonist administration in normal and high-fat diet C57BL6 mice," Advanced Biomedical Research, vol. 5, no. 1, p. 165, 2016.

[6] R. J. Steagall, C. R. Daniels, S. Dalal, W. L. Joyner, M. Singh, and K. Singh, "Extracellular ubiquitin increases expression of angiogenic molecules and stimulates angiogenesis in cardiac microvascular endothelial cells," Microcirculation, vol. 21, no. 4, pp. 324-332, 2014.

[7] M. Shibuya, "Vascular endothelial growth factor and its receptor system: physiological functions in angiogenesis and pathological roles in various diseases," Journal of Biochemistry, vol. 153, no. 1, pp. 13-19, 2013.

[8] F. Yang, W. Zhang, D. Li, and Q. Zhan, "Gadd45a suppresses tumor angiogenesis via inhibition of the mTOR/STAT3 protein pathway," Journal of Biological Chemistry, vol. 288, no. 9, pp. 6552-6560, 2013.

[9] N. Prevete, F. Liotti, G. Marone, R. M. Melillo, and A. de Paulis, "Formyl peptide receptors at the interface of inflammation, angiogenesis and tumor growth," Pharmacological Research, vol. 102, pp. 184-191, 2015.

[10] R. Thom, G. C. Rowe, C. Jang, A. Safdar, and Z. Arany, "Hypoxic induction of vascular endothelial growth factor (VEGF) and angiogenesis in muscle by truncated peroxisome proliferator-activated receptor $\gamma$ coactivator (PGC)-1 $\alpha$," Journal of Biological Chemistry, vol. 289, no. 13, pp. 88108817, 2014.

[11] M. Alasvand, B. Rashidi, S. H. Javanmard, M. M. Akhavan, and M. Khazaei, "Effect of blocking of neuropeptide Y Y2 receptor on tumor angiogenesis and progression in normal and diet-induced obese C57BL/6 mice," Global Journal of Health Science, vol. 7, no. 7, pp. 69-78, 2015.

[12] J. Karar and A. Maity, "PI3K/AKT/mTOR pathway in angiogenesis," Frontiers in Molecular Neuroscience, vol. 4, p. 51, 2011.

[13] A. Zimna and M. Kurpisz, "Hypoxia-inducible factor-1 in physiological and pathophysiological angiogenesis: applications and therapies," BioMed Research International, vol. 2015, Article ID 549412, 13 pages, 2015.

[14] R. Malhotra, D. W. Tyson, H. M. Rosevear, and F. C. Brosius, "Hypoxia-inducible factor-1alpha is a critical mediator of hypoxia induced apoptosis in cardiac $\mathrm{H} 9 \mathrm{c} 2$ and kidney epithelial HK-2 cells," BMC Cardiovascular Disorders, vol. 8, no. 1, pp. 1-11, 2008.

[15] B. Z. Olenyuk, G.-J. Zhang, J. M. Klco, N. G. Nickols, W. G. Kaelin, and P. B. Dervan, "Inhibition of vascular endothelial growth factor with a sequence-specific hypoxia response element antagonist," Proceedings of the National Academy of Sciences of the United States of America, vol. 101, no. 48, pp. 16768-16773, 2004.

[16] A. Ahluwalia and A. S. Tarnawski, "Critical role of hypoxia sensor-HIF- $1 \alpha$ in VEGF gene activation. Implications for angiogenesis and tissue injury healing," Current Medicinal Chemistry, vol. 19, no. 1, pp. 90-97, 2012.

[17] M. Litwin, A. Radwańska, M. Paprocka et al., “The role of FGF2 in migration and tubulogenesis of endothelial progenitor cells in relation to pro-angiogenic growth factor production," Molecular and Cellular Biochemistry, vol. 410, no. 1-2, pp. 131-142, 2015.

[18] I. Ghanem, M. E. Riveiro, V. Paradis, S. Faivre, P. de Parga, and E. Raymond, "Insights on the CXCL12-CXCR4 axis in hepatocellular carcinoma carcinogenesis," American Journal of Translational Research, vol. 6, no. 4, pp. 340-352, 2014.

[19] J. Welti, S. Loges, S. Dimmeler, and P. Carmeliet, "Recent molecular discoveries in angiogenesis and antiangiogenic therapies in cancer," The Journal of Clinical Investigation, vol. 123, no. 8, pp. 3190-3200, 2013.

[20] W. A. Barton, A. C. Dalton, T. C. M. Seegar, J. P. Himanen, and D. B. Nikolov, "Tie 2 and Eph receptor tyrosine kinase activation and signaling," Cold Spring Harbor Perspectives in Biology, vol. 6, no. 3, 2014.

[21] D. I. R. Holmes and I. Zachary, "The vascular endothelial growth factor (VEGF) family: angiogenic factors in health and disease," Genome Biology, vol. 6, no. 2, p. 209, 2005.

[22] M. Shibuya, "Vascular endothelial growth factor (VEGF) and its receptor (VEGFR) signaling in angiogenesis: a crucial target for anti- and pro-angiogenic therapies," Genes \& Cancer, vol. 2, no. 12, pp. 1097-1105, 2012.

[23] S. Koch and L. Claesson-Welsh, "Signal transduction by vascular endothelial growth factor receptors," Cold Spring Harbor Perspectives in Medicine, vol. 2, no. 7, article a006502, 2012.

[24] S. Germain, C. Monnot, L. Muller, and A. Eichmann, "Hypoxia-driven angiogenesis: role of tip cells and extracellular matrix scaffolding," Current Opinion in Hematology, vol. 17, no. 3, pp. 245-251, 2010.

[25] S. Koch, S. Tugues, X. Li, L. Gualandi, and L. Claesson-Welsh, "Signal transduction by vascular endothelial growth factor receptors," Biochemical Journal, vol. 437, no. 2, pp. 169183, 2011.

[26] T. C. Lobo, L. Magalhães, L. Cardeal, R. Giordano, A. Baldwin, and D. Bassères, "IKK $\beta$ is a potential antiangiogenic therapeutic target in KRAS-induced lung cancer," Cancer Research, vol. 75, Supplement 15, p. 1379, 2015.

[27] N. T. Devika and B. M. J. Ali, “Analysing calcium dependent and independent regulation of eNOS in endothelium triggered by extracellular signalling events," Molecular BioSystems, vol. 9, no. 11, pp. 2653-2664, 2013.

[28] H. F. Dvorak, L. F. Brown, M. Detmar, and A. M. Dvorak, "Vascular permeability factor/vascular endothelial growth factor, microvascular hyperpermeability, and angiogenesis," The American Journal of Pathology, vol. 146, no. 5, pp. 1029-1039, 1995. 
[29] L. Claesson-Welsh and M. Welsh, "VEGFA and tumour angiogenesis," Journal of Internal Medicine, vol. 273, no. 2, pp. 114-127, 2013.

[30] A. S. Chung, J. Lee, and N. Ferrara, "Targeting the tumour vasculature: insights from physiological angiogenesis," Nature Reviews Cancer, vol. 10, no. 7, pp. 505-514, 2010.

[31] G. Zhuang, X. Wu, Z. Jiang et al., "Tumour-secreted miR-9 promotes endothelial cell migration and angiogenesis by activating the JAK-STAT pathway," The EMBO Journal, vol. 31, no. 17, pp. 3513-3523, 2012.

[32] M. Teodorczyk, N. Dudvarski Stanković, F. Bicker, and H. M. Schmidt, "VEGF and Notch signaling in angiogenesis," in Endothelial Signaling in Development and Disease, H. H. M. Schmidt and S. Liebner, Eds., pp. 3-46, Springer New York, New York, NY, USA, 2015.

[33] E. Rabizadeh, I. Cherny, D. Lederfein et al., "The cellmembrane prothrombinase, fibrinogen-like protein 2 , promotes angiogenesis and tumor development," Thrombosis Research, vol. 136, no. 1, pp. 118-124, 2015.

[34] M. Belting, J. Ahamed, and W. Ruf, "Signaling of the tissue factor coagulation pathway in angiogenesis and cancer," Arteriosclerosis, Thrombosis, and Vascular Biology, vol. 25, no. 8, pp. 1545-1550, 2005.

[35] D. Ribatti and V. Djonov, "Intussusceptive microvascular growth in tumors," Cancer Letters, vol. 316, no. 2, pp. 126$131,2012$.

[36] L. M. Willis, A. B. El-Remessy, P. R. Somanath, D. L. Deremer, and S. C. Fagan, "Angiotensin receptor blockers and angiogenesis: clinical and experimental evidence," Clinical Science, vol. 120, no. 8, pp. 307-319, 2011.

[37] D. Bouis, Y. Kusumanto, C. Meijer, N. H. Mulder, and G. A. Hospers, "A review on pro- and anti-angiogenic factors as targets of clinical intervention," Pharmacological Research, vol. 53, no. 2, pp. 89-103, 2006.

[38] L. A. Sullivan and R. A. Brekken, "The VEGF family in cancer and antibody-based strategies for their inhibition," $m A b s$, vol. 2, no. 2, pp. 165-175, 2010.

[39] G. W. Prager, M. Poettler, M. Unseld, and C. C. Zielinski, "Angiogenesis in cancer: anti-VEGF escape mechanisms," Translational Lung Cancer Research, vol. 1, no. 1, pp. 1425, 2012.

[40] A. M. E. Abdalla, L. Xiao, M. W. Ullah, M. Yu, C. Ouyang, and G. Yang, "Current challenges of cancer anti-angiogenic therapy and the promise of nanotherapeutics," Theranostics, vol. 8, no. 2, pp. 533-548, 2018.

[41] J. J. Vredenburgh, A. Desjardins, J. E. Herndon II et al., "Bevacizumab plus irinotecan in recurrent glioblastoma multiforme," Journal of Clinical Oncology, vol. 25, no. 30, pp. 4722-4729, 2007.

[42] R. K. Jain, "Normalization of tumor vasculature: an emerging concept in antiangiogenic therapy," Science, vol. 307, no. 5706 , pp. 58-62, 2005.

[43] C. N. Sternberg, I. D. Davis, J. Mardiak et al., "Pazopanib in locally advanced or metastatic renal cell carcinoma: results of a randomized phase III trial," Journal of Clinical Oncology, vol. 28, no. 6, pp. 1061-1068, 2010.

[44] B. Escudier, T. Eisen, W. M. Stadler et al., "Sorafenib in advanced clear-cell renal-cell carcinoma," The New England Journal of Medicine, vol. 356, no. 2, pp. 125134, 2007.
[45] J. M. Llovet, S. Ricci, V. Mazzaferro et al., "Sorafenib in advanced hepatocellular carcinoma," The New England Journal of Medicine, vol. 359, no. 4, pp. 378-390, 2008.

[46] F. Elice and F. Rodeghiero, "Side effects of anti-angiogenic drugs," Thrombosis Research, vol. 129, Supplement 1, pp. S50-S53, 2012.

[47] E. Maj, D. Papiernik, and J. Wietrzyk, “Antiangiogenic cancer treatment: the great discovery and greater complexity (review)," International Journal of Oncology, vol. 49, no. 5, pp. 1773-1784, 2016.

[48] Z. Wang, C. Dabrosin, X. Yin et al., "Broad targeting of angiogenesis for cancer prevention and therapy," Seminars in Cancer Biology, vol. 35, pp. S224-S243, 2015.

[49] J. Jiang and C. Hu, "Evodiamine: a novel anti-cancer alkaloid from Evodia rutaecarpa," Molecules, vol. 14, no. 5, article 1852, 1859 pages, 2009.

[50] S. M. Sagar, D. Yance, and R. K. Wong, "Natural health products that inhibit angiogenesis: a potential source for investigational new agents to treat cancer-part 1," Current Oncology, vol. 13, no. 1, pp. 14-26, 2006.

[51] M. Singh, P. Singh, and Y. Shukla, "New strategies in cancer chemoprevention by phytochemicals," Frontiers in Bioscience, vol. E4, no. 1, pp. 426-452, 2012.

[52] N. Saki, S. Abroun, M. Soleimani et al., "Involvement of microRNA in T-cell differentiation and malignancy," International Journal of Hematology-Oncology and Stem Cell Research, vol. 9, no. 1, pp. 33-49, 2015.

[53] M. Jiao, H. Z. You, X. Y. Yang et al., "Circulating microRNA signature for the diagnosis of childhood dilated cardiomyopathy," Scientific Reports, vol. 8, no. 1, p. 724, 2018.

[54] N. J. Leeper and J. P. Cooke, "MicroRNA and mechanisms of impaired angiogenesis in diabetes mellitus," Circulation, vol. 123, no. 3, pp. 236-238, 2011.

[55] E. Hergenreider, S. Heydt, K. Tréguer et al., "Atheroprotective communication between endothelial cells and smooth muscle cells through miRNAs," Nature Cell Biology, vol. 14, no. 3, pp. 249-256, 2012.

[56] L. T. H. Dang, N. D. Lawson, and J. E. Fish, "MicroRNA control of vascular endothelial growth factor signaling output during vascular development," Arteriosclerosis, Thrombosis, and Vascular Biology, vol. 33, no. 2, pp. 193-200, 2013.

[57] Y. Suarez and W. C. Sessa, "MicroRNAs as novel regulators of angiogenesis," Circulation Research, vol. 104, no. 4, pp. 442 454, 2009.

[58] J. Chen and D. Z. Wang, "MicroRNAs in cardiovascular development," Journal of Molecular and Cellular Cardiology, vol. 52, no. 5, pp. 949-957, 2012.

[59] A. Tiwari, B. Mukherjee, and M. Dixit, "MicroRNA key to angiogenesis regulation: MiRNA biology and therapy," Current Cancer Drug Targets, vol. 18, no. 3, pp. 266-277, 2018.

[60] S. Gallach, S. Calabuig-Fariñas, E. Jantus-Lewintre, and C. Camps, "MicroRNAs: promising new antiangiogenic targets in cancer," BioMed Research International, vol. 2014, Article ID 878450, 14 pages, 2014.

[61] S. H. Chang and T. Hla, "Gene regulation by RNA binding proteins and microRNAs in angiogenesis," Trends in Molecular Medicine, vol. 17, no. 11, pp. 650-658, 2011.

[62] S. Wang and E. N. Olson, "AngiomiRs-key regulators of angiogenesis," Current Opinion in Genetics \& Development, vol. 19, no. 3, pp. 205-211, 2009. 
[63] C. Urbich, A. Kuehbacher, and S. Dimmeler, "Role of microRNAs in vascular diseases, inflammation, and angiogenesis," Cardiovascular Research, vol. 79, no. 4, pp. 581-588, 2008.

[64] W.-X. Chen, Q. Hu, M.-T. Qiu et al., "miR-221/222: promising biomarkers for breast cancer," Tumor Biology, vol. 34, no. 3, pp. 1361-1370, 2013.

[65] X. Zhao, H. Q. Liu, J. Li, and X. L. Liu, "Endothelial progenitor cells promote tumor growth and progression by enhancing new vessel formation," Oncology Letters, vol. 12, no. 2, pp. 793-799, 2016.

[66] M. Marcola and C. E. Rodrigues, "Endothelial progenitor cells in tumor angiogenesis: another brick in the wall," Stem Cells International, vol. 2015, Article ID 832649, 10 pages, 2015.

[67] T. Zhao, J. Li, and A. F. Chen, "MicroRNA-34a induces endothelial progenitor cell senescence and impedes its angiogenesis via suppressing silent information regulator 1," American Journal of Physiology-Endocrinology and Metabolism, vol. 299, no. 1, pp. E110-E116, 2010.

[68] J. J. Chen and S. H. Zhou, "Mesenchymal stem cells overexpressing MiR-126 enhance ischemic angiogenesis via the AKT/ERK-related pathway," Cardiology Journal, vol. 18, no. 6, pp. 675-681, 2011.

[69] J. E. Fish, M. M. Santoro, S. U. Morton et al., "miR-126 regulates angiogenic signaling and vascular integrity," Developmental Cell, vol. 15, no. 2, pp. 272-284, 2008.

[70] T. Sasahira, M. Kurihara, U. K. Bhawal et al., "Downregulation of miR-126 induces angiogenesis and lymphangiogenesis by activation of VEGF-A in oral cancer," British Journal of Cancer, vol. 107, no. 4, pp. 700-706, 2012.

[71] L. Fang, Z. Deng, T. Shatseva et al., "MicroRNA miR-93 promotes tumor growth and angiogenesis by targeting integrin$\beta 8$," Oncogene, vol. 30, no. 7, pp. 806-821, 2011.

[72] L. Fang, W. W. Du, W. Yang et al., "MiR-93 enhances angiogenesis and metastasis by targeting LATS2," Cell Cycle, vol. 11, no. 23, pp. 4352-4365, 2012.

[73] S. Chen, Y. Xue, X. Wu et al., "Global microRNA depletion suppresses tumor angiogenesis," Genes \& Development, vol. 28, no. 10, pp. 1054-1067, 2014.

[74] S. Landskroner-Eiger, I. Moneke, and W. C. Sessa, "MiRNAs as modulators of angiogenesis," Cold Spring Harbor Perspectives in Medicine, vol. 3, no. 2, article a006643, 2013.

[75] M. Yamakuchi, C. D. Lotterman, C. Bao et al., "P53-induced microRNA-107 inhibits HIF-1 and tumor angiogenesis," Proceedings of the National Academy of Sciences of the United States of America, vol. 107, no. 14, pp. 6334-6339, 2010.

[76] S. Anand, B. K. Majeti, L. M. Acevedo et al., "MicroRNA-132mediated loss of p120RasGAP activates the endothelium to facilitate pathological angiogenesis," Nature Medicine, vol. 16, no. 8, pp. 909-914, 2010.

[77] G. Ghosh, I. V. Subramanian, N. Adhikari et al., "Hypoxiainduced microRNA-424 expression in human endothelial cells regulates HIF- $\alpha$ isoforms and promotes angiogenesis," The Journal of Clinical Investigation, vol. 120, no. 11, pp. 4141-4154, 2010.

[78] J. H. Fang, H. C. Zhou, C. Zeng et al., "MicroRNA-29b suppresses tumor angiogenesis, invasion, and metastasis by regulating matrix metalloproteinase 2 expression," Hepatology, vol. 54, no. 5, pp. 1729-1740, 2011.

[79] S. T. Cha, P. S. Chen, G. Johansson et al., "MicroRNA519c suppresses hypoxia-inducible factor- $1 \alpha$ expression and tumor angiogenesis," Cancer Research, vol. 70, no. 7, pp. 2675-2685, 2010.

[80] F. Liu, Y. L. Lou, J. Wu et al., "Upregulation of microRNA-210 regulates renal angiogenesis mediated by activation of VEGF signaling pathway under ischemia/perfusion injury in vivo and in vitro," Kidney and Blood Pressure Research, vol. 35, no. 3, pp. 182-191, 2012.

[81] W. Kong, L. He, E. J. Richards et al., "Upregulation of miRNA-155 promotes tumour angiogenesis by targeting VHL and is associated with poor prognosis and triplenegative breast cancer," Oncogene, vol. 33, no. 6, pp. 679-689, 2014.

[82] R. Wang, N. Zhao, S. Li et al., "MicroRNA-195 suppresses angiogenesis and metastasis of hepatocellular carcinoma by inhibiting the expression of VEGF, VAV2, and CDC42," Hepatology, vol. 58, no. 2, pp. 642-653, 2013.

[83] H. Zhang, J. Pu, T. Qi et al., "MicroRNA-145 inhibits the growth, invasion, metastasis and angiogenesis of neuroblastoma cells through targeting hypoxia-inducible factor 2 alpha," Oncogene, vol. 33, no. 3, pp. 387-397, 2014.

[84] X. Yang, X. F. Zhang, X. Lu et al., "MicroRNA-26a suppresses angiogenesis in human hepatocellular carcinoma by targeting hepatocyte growth factor-cMet pathway," Hepatology, vol. 59, no. 5, pp. 1874-1885, 2014.

[85] T. C. Shih, Y. J. Tien, C. J. Wen et al., "MicroRNA-214 downregulation contributes to tumor angiogenesis by inducing secretion of the hepatoma-derived growth factor in human hepatoma," Journal of Hepatology, vol. 57, no. 3, pp. 584$591,2012$.

[86] M. Meloni, M. Marchetti, K. Garner et al., "Local inhibition of microRNA-24 improves reparative angiogenesis and left ventricle remodeling and function in mice with myocardial infarction," Molecular Therapy, vol. 21, no. 7, pp. 1390$1402,2013$.

[87] J. Wang, Y. Wang, Y. Wang, Y. Ma, Y. Lan, and X. Yang, "Transforming growth factor $\beta$-regulated microRNA-29a promotes angiogenesis through targeting the phosphatase and tensin homolog in endothelium," Journal of Biological Chemistry, vol. 288, no. 15, pp. 10418-10426, 2013.

[88] J. Ye, X. Wu, D. Wu et al., "miRNA-27b targets vascular endothelial growth factor $\mathrm{C}$ to inhibit tumor progression and angiogenesis in colorectal cancer," PLoS One, vol. 8, no. 4, article e60687, 2013.

[89] B. Zhou, R. Ma, W. Si et al., "MicroRNA-503 targets FGF2 and VEGFA and inhibits tumor angiogenesis and growth," Cancer Letters, vol. 333, no. 2, pp. 159-169, 2013.

[90] X. Qian, J. Yu, Y. Yin et al., "MicroRNA-143 inhibits tumor growth and angiogenesis and sensitizes chemosensitivity to oxaliplatin in colorectal cancers," Cell Cycle, vol. 12, no. 9, pp. 1385-1394, 2013.

[91] J. K. Seok, S. H. Lee, M. J. Kim, and Y. M. Lee, "MicroRNA382 induced by HIF- $1 \alpha$ is an angiogenic miR targeting the tumor suppressor phosphatase and tensin homolog," Nucleic Acids Research, vol. 42, no. 12, pp. 8062-8072, 2014.

[92] L. Zeng, X. He, Y. Wang et al., "MicroRNA-210 overexpression induces angiogenesis and neurogenesis in the normal adult mouse brain," Gene Therapy, vol. 21, no. 1, pp. 37-43, 2014.

[93] T. He, F. Qi, L. Jia et al., "MicroRNA-542-3p inhibits tumour angiogenesis by targeting angiopoietin-2," The Journal of Pathology, vol. 232, no. 5, pp. 499-508, 2014. 
[94] A. van Mil, S. Grundmann, M. J. Goumans et al., "MicroRNA-214 inhibits angiogenesis by targeting Quaking and reducing angiogenic growth factor release," Cardiovascular Research, vol. 93, no. 4, pp. 655-665, 2012.

[95] L. A. Shehadeh, S. Sharma, M. Pessanha et al., "MicroRNA-20a constrains p300-driven myocardial angiogenic transcription by direct targeting of p300," PLoS One, vol. 8, no. 11, article e79133, 2013.

[96] K. J. Yin, K. Olsen, M. Hamblin, J. Zhang, S. P. Schwendeman, and Y. E. Chen, "Vascular endothelial cell-specific microRNA-15a inhibits angiogenesis in hindlimb ischemia," Journal of Biological Chemistry, vol. 287, no. 32, pp. 27055-27064, 2012.

[97] E. W. Newcomb, Y. Lukyanov, T. Schnee, M. A. Ali, L. Lan, and D. Zagzag, "Noscapine inhibits hypoxiamediated HIF- $1 \alpha$ expression andangiogenesis in vitro: a novel function for an old drug," International Journal of Oncology, vol. 28, no. 5, pp. 1121-1130, 2006.

[98] E. W. Newcomb, Y. Lukyanov, M. Alonso-Basanta et al., "Antiangiogenic effects of noscapine enhance radioresponse for GL261 tumors," International Journal of Radiation Oncology Biology Physics, vol. 71, no. 5, pp. 1477-1484, 2008.

[99] B. Sung, K. S. Ahn, and B. B. Aggarwal, "Noscapine, a benzylisoquinoline alkaloid, sensitizes leukemic cells to chemotherapeutic agents and cytokines by modulating the NF- $\kappa \mathrm{B}$ signaling pathway," Cancer Research, vol. 70, no. 8, pp. 3259-3268, 2010.

[100] M. B. Chougule, A. R. Patel, T. Jackson, and M. Singh, "Antitumor activity of noscapine in combination with doxorubicin in triple negative breast cancer," PLoS One, vol. 6, no. 3, article e17733, 2011.

[101] W. Su, L. Huang, Q. Ao et al., "Noscapine sensitizes chemoresistant ovarian cancer cells to cisplatin through inhibition of HIF-1 $\alpha$," Cancer Letters, vol. 305, no. 1, pp. 94-99, 2011.

[102] S. Lin, S. C. Tsai, C. C. Lee, B. W. Wang, J. Y. Liou, and K. G. Shyu, "Berberine inhibits HIF-1 $\alpha$ expression via enhanced proteolysis," Molecular Pharmacology, vol. 66, no. 3, pp. 612-619, 2004.

[103] L. Lin, Z. Na, G. Chunhua, Z. Xiaoyi, and F. Yimin, "Effects of berberine on apoptosis and expression of apoptosis-related proteins in HeLa cells," Chinese Traditional and Herbal Drug, vol. 39, no. 2, pp. 244-247, 2008.

[104] J.-L. Gao, J.-M. Shi, S. M.-Y. Lee, Q.-W. Zhang, and Y.T. Wang, "Angiogenic pathway inhibition of Corydalis yanhusuo and berberine in human umbilical vein endothelial cells," Oncology Research, vol. 17, no. 11, pp. 519-526, 2009.

[105] Y. T. Ho, C. C. Lu, J. S. Yang et al., "Berberine induced apoptosis via promoting the expression of caspase- $8,-9$ and -3 , apoptosis-inducing factor and endonuclease G in SCC-4 human tongue squamous carcinoma cancer cells," Anticancer Research, vol. 29, no. 10, pp. 4063-4070, 2009.

[106] S. Jie, H. Li, Y. Tian et al., "Berberine inhibits angiogenic potential of Hep G2 cell line through VEGF downregulation in vitro," Journal of Gastroenterology and Hepatology, vol. 26, no. 1, pp. 179-185, 2011.

[107] T. P. Hamsa and G. Kuttan, "Antiangiogenic activity of berberine is mediated through the downregulation of hypoxiainducible factor-1, VEGF, and proinflammatory mediators," Drug and Chemical Toxicology, vol. 35, no. 1, pp. 57-70, 2012.

[108] W. Ruhua, "Study of berberine on the 125I particle in radiosensitization and its effects of angiogenesis on nude mice hepatocellular carcinoma," Chinese Journal of Surgical Oncology, vol. 4, no. 5, pp. 275-278, 2012.

[109] S. Kim, S. J. Oh, J. Lee et al., "Berberine suppresses TPA-induced fibronectin expression through the inhibition of VEGF secretion in breast cancer cells," Cellular Physiology and Biochemistry, vol. 32, no. 5, pp. 15411550, 2013.

[110] Y.-f. Wang, S.-d. Ke, and X.-l. Tao, "Effects of berberine on the expression of vascular endothelial growth factor in HepG2 cells," Chinese Journal of Integrated Traditional and Western Medicine on Digestion, vol. 21, no. 3, pp. 143-145, 2013.

[111] L. Fu, W. Chen, W. Guo et al., "Berberine targets AP2/hTERT, NF- $\kappa$ B/COX-2, HIF- $1 \alpha /$ VEGF and cytochromec/caspase signaling to suppress human cancer cell growth," PLoS One, vol. 8, no. 7, article e69240, 2013.

[112] S. C. Chu, C. C. Yu, L. S. Hsu, K. S. Chen, M. Y. Su, and P. N. Chen, "Berberine reverses epithelial-to-mesenchymal transition and inhibits metastasis and tumor-induced angiogenesis in human cervical cancer cells," Molecular Pharmacology, vol. 86, no. 6, pp. 609-623, 2014.

[113] C. M. Tsang, K. C. P. Cheung, Y. C. Cheung et al., "Berberine suppresses Id- 1 expression and inhibits the growth and development of lung metastases in hepatocellular carcinoma," Biochimica et Biophysica Acta (BBA) - Molecular Basis of Disease, vol. 1852, no. 3, pp. 541-551, 2015.

[114] F. Bianco, G. Basini, and F. Grasselli, "The plant alkaloid sanguinarine affects swine granulosa cell activity," Reproductive Toxicology, vol. 21, no. 3, pp. 335-340, 2006.

[115] G. Basini, S. Bussolati, S. E. Santini, and F. Grasselli, "Sanguinarine inhibits VEGF-induced angiogenesis in a fibrin gel matrix," Biofactors, vol. 29, no. 1, pp. 11-18, 2007.

[116] G. Basini, S. E. Santini, S. Bussolati, and F. Grasselli, "The plant alkaloid sanguinarine is a potential inhibitor of follicular angiogenesis," Journal of Reproduction and Development, vol. 53, no. 3, pp. 573-579, 2007.

[117] G. Basini, S. E. Santini, S. Bussolati, and F. Grasselli, "Sanguinarine inhibits VEGF-induced Akt phosphorylation," Annals of the New York Academy of Sciences, vol. 1095, no. 1, pp. 371-376, 2007.

[118] I. De Stefano, G. Raspaglio, G. F. Zannoni et al., “Antiproliferative and antiangiogenic effects of the benzophenanthridine alkaloid sanguinarine in melanoma," Biochemical Pharmacology, vol. 78, no. 11, pp. 1374-1381, 2009.

[119] J. Y. Xu, Q. H. Meng, Y. Chong et al., "Sanguinarine is a novel VEGF inhibitor involved in the suppression of angiogenesis and cell migration," Molecular and Clinical Oncology, vol. 1, no. 2, pp. 331-336, 2013.

[120] X. Z. Dong, M. Zhang, K. Wang et al., "Sanguinarine inhibits vascular endothelial growth factor release by generation of reactive oxygen species in MCF-7 human mammary adenocarcinoma cells," BioMed Research International, vol. 2013, Article ID 517698, 8 pages, 2013.

[121] X. H. Du, S. Huang, H. R. Feng, L. L. Chen, S. Yang, and L. F. $\mathrm{Lu}$, "Inhibition of sanguinarine on S180 subcutaneously implanted tumors in mice," Zhong Yao Cai, vol. 37, no. 10, pp. 1830-1833, 2014.

[122] C. K. Singh, S. Kaur, J. George et al., "Molecular signatures of sanguinarine in human pancreatic cancer cells: a large scale label-free comparative proteomics approach," Oncotarget, vol. 6, no. 12, pp. 10335-10348, 2015. 
[123] X. J. Li, P. Y. K. Yue, W. Y. Ha et al., "Effect of sinomenine on gene expression of the IL- $1 \beta$-activated human synovial sarcoma," Life Sciences, vol. 79, no. 7, pp. 665-673, 2006.

[124] T. Xie, H. Y. Ren, H. Q. Lin et al., "Sinomenine prevents metastasis of human osteosarcoma cells via $S$ phase arrest and suppression of tumor-related neovascularization and osteolysis through the CXCR4-STAT3 pathway," International Journal of Oncology, vol. 48, no. 5, pp. 2098-2112, 2016.

[125] Y. Zhang, L. He, L. Meng, W. Luo, and X. Xu, "Suppression of tumor-induced angiogenesis by taspine isolated from Radix et Rhizoma Leonticis and its mechanism of action in vitro," Cancer Letters, vol. 262, no. 1, pp. 103113, 2008.

[126] J. Zhao, L. Zhao, W. Chen, L. He, and X. Li, “Taspine downregulates VEGF expression and inhibits proliferation of vascular endothelial cells through PI3 kinase and MAP kinase signaling pathways," Biomedicine \& Pharmacotherapy, vol. 62, no. 6, pp. 383-389, 2008.

[127] J. Zhang, Y. Zhang, Y. Shan, N. Li, W. Ma, and L. He, "Synthesis and preliminary biological evaluation of novel taspine derivatives as anticancer agents," European Journal of Medicinal Chemistry, vol. 45, no. 7, pp. 2798-2805, 2010.

[128] Y. Zhang, J. Zhang, B. Dai, N. Wang, and L. He, “Anti-proliferative and apoptotic effects of the novel taspine derivative tas41 in the Caco-2 cell line," Environmental Toxicology and Pharmacology, vol. 31, no. 3, pp. 406-415, 2011.

[129] Y. M. Zhang, N. Wang, B. L. Dai, and L. C. He, "Effect of taspine derivatives on human liver cancer SMMC 7721," Zhong Yao Cai, vol. 34, no. 7, pp. 1094-1097, 2011.

[130] Y. M. Zhang, B. L. Dai, L. Zheng et al., "A novel angiogenesis inhibitor impairs lovo cell survival via targeting against human VEGFR and its signaling pathway of phosphorylation," Cell Death \& Disease, vol. 3, no. 10, article e406, 2012.

[131] L. Zheng, X. He, W. Ma, B. Dai, Y. Zhan, and Y. Zhang, “Ta 1722, an anti-angiogenesis inhibitor targeted on VEGFR-2 against human hepatoma," Biomedicine \& Pharmacotherapy, vol. 66, no. 7, pp. 499-505, 2012.

[132] W. Lu, B. Dai, W. Ma, and Y. Zhang, "A novel taspine analog, HMQ1611, inhibits growth of non-small cell lung cancer by inhibiting angiogenesis," Oncology Letters, vol. 4, no. 5, pp. 1109-1113, 2012.

[133] S. Kobayashi, K. Inaba, I. Kimura, and M. Kimura, "Inhibitory effects of tetrandrine on angiogenesis in adjuvantinduced chronic inflammation and tube formation of vascular endothelial cells," Biological \& Pharmaceutical Bulletin, vol. 21, no. 4, pp. 346-349, 1998.

[134] Y. Chen, J. C. Chen, and S. H. Tseng, "Tetrandrine suppresses tumor growth and angiogenesis of gliomas in rats," International Journal of Cancer, vol. 124, no. 10, pp. 2260-2269, 2009.

[135] G. Sun, G. Su, Y. Zu, M. Zhang, and Q. Shi, "Effect of tetrandrine on the expressions of hypoxia-inducible factor-1 and vascular endothelial growth factor in neovascularized cornea," African Journal of Pharmacy and Pharmacology, vol. 5, no. 9, pp. 1223-1227, 2011.

[136] J. L. Gao, X. Ji, T. C. He et al., “Tetrandrine suppresses cancer angiogenesis and metastasis in 4T1 tumor bearing mice," Evidence-Based Complementary and Alternative Medicine, vol. 2013, Article ID 265061, 12 pages, 2013.
[137] X. Qian, B. Yan, X. Zhou et al., "Synergistic antiangiogenic activity of tetrandrine combined with Endostar on the human umbilical vein endothelial cell model," Cancer Biotherapy and Radiopharmaceuticals, vol. 28, no. 5, pp. 385-390, 2013.

[138] W. Xiao, Y. Jiang, Q. Men et al., “Tetrandrine induces G1/S cell cycle arrest through the ROS/Akt pathway in EOMA cells and inhibits angiogenesis in vivo," International Journal of Oncology, vol. 46, no. 1, pp. 360-368, 2015.

[139] S. S. Agrawal, S. Saraswati, R. Mathur, and M. Pandey, "Brucine, a plant derived alkaloid inhibits inflammatory angiogenesis in a murine sponge model," Biomedicine \& Preventive Nutrition, vol. 1, no. 3, pp. 180-185, 2011.

[140] S. S. Agrawal, S. Saraswati, R. Mathur, and M. Pandey, "Cytotoxic and antitumor effects of brucine on Ehrlich ascites tumor and human cancer cell line," Life Sciences, vol. 89, no. 5-6, pp. 147-158, 2011.

[141] P. Li, M. Zhang, W. J. Ma, X. Sun, and F. P. Jin, "Effects of brucine on vascular endothelial growth factor expression and microvessel density in a nude mouse model of bone metastasis due to breast cancer," Chinese Journal of Integrative Medicine, vol. 18, no. 8, pp. 605-609, 2012.

[142] G. Shu, X. Mi, J. Cai et al., "Brucine, an alkaloid from seeds of Strychnos nux-vomica Linn., represses hepatocellular carcinoma cell migration and metastasis: the role of hypoxia inducible factor 1 pathway," Toxicology Letters, vol. 222, no. 2, pp. 91-101, 2013.

[143] W. Luo, X. Wang, L. Zheng et al., "Brucine suppresses colon cancer cells growth via mediating KDR signalling pathway," Journal of Cellular and Molecular Medicine, vol. 17, no. 10, pp. 1316-1324, 2013.

[144] L. Zheng, X. Wang, W. Luo, Y. Zhan, and Y. Zhang, "Brucine, an effective natural compound derived from nuxvomica, induces G1 phase arrest and apoptosis in LoVo cells," Food and Chemical Toxicology, vol. 58, pp. 332339, 2013.

[145] X. Kechun and M. Zjauma, "Experimental study of brucine by percutaneous administration to inhibit expressions of COX-2 mRNA and VEGF mRNA in plant tumor of breast cancer," Acta Universitatis Medicinalis Anhui, no. 1, p. 4, 2013.

[146] K. G. Shyu, S. Lin, C. C. Lee et al., "Evodiamine inhibits in vitro angiogenesis: implication for antitumorgenicity," Life Sciences, vol. 78, no. 19, pp. 2234-2243, 2006.

[147] P. S. Wang, C.-H. Yu, and J.-C. Su, "Effects of evodiamine on the expression and secretion of vascular endothelial growth factor in human breast cancer cells," Biology of Reproduction, vol. 78, Supplement 1, p. 215, 2008.

[148] J. Huang, Z.-H. Chen, C.-M. Ren et al., “Antiproliferation effect of evodiamine in human colon cancer cells is associated with IGF-1/HIF-1 $\alpha$ downregulation," Oncology Reports, vol. 34, no. 6, pp. 3203-3211, 2015.

[149] X. J. Ye and M. F. Lin, "Homoharringtonine induces apoptosis of endothelium and down-regulates VEGF expression of K562 cells," Journal of Zhejiang University Science, vol. 5, no. 2, pp. 230-234, 2004.

[150] J. Fei and H. Zhang, "Vascular endothelial growth factor antisense oligodeoxynucleotide enhance drug-sensitivity of myeloid leukemia cells to homoharringtonine," Zhonghua Xue Ye Xue Za Zhi, vol. 26, no. 1, pp. 27-30, 2005.

[151] B. Z. Li, W. Z. Zhuang, P. Chen, and J. X. Fu, "Effects of RNA interference targeting hypoxia-inducible factor- $1 \alpha$ 
(HIF-1 $\alpha$ ) on chemosensitivity of leukemia K562 cells towards homoharringtonine," Ai Zheng, vol. 27, no. 7, pp. 723-728, 2008.

[152] P. Yu, Q. Liu, K. Liu, K. Yagasaki, E. Wu, and G. Zhang, "Matrine suppresses breast cancer cell proliferation and invasion via VEGF-Akt-NF- $\kappa \mathrm{B}$ signaling," Cytotechnology, vol. 59, no. 3, pp. 219-229, 2009.

[153] D. J. Gross, I. Reibstein, L. Weiss et al., "Treatment with halofuginone results in marked growth inhibition of a von Hippel-Lindau pheochromocytoma in vivo," Clinical Cancer Research, vol. 9, 10, Part 1, pp. 3788-3793, 2003.

[154] R. Abramovitch, A. Itzik, H. Harel, A. Nagler, I. Vlodavsky, and T. Siegal, "Halofuginone inhibits angiogenesis and growth in implanted metastatic rat brain tumor model-an MRI study," Neoplasia, vol. 6, no. 5, pp. 480-489, 2004.

[155] K. O. Yee, C. M. Connolly, M. Pines, and J. Lawler, "Halofuginone inhibits tumor growth in the polyoma middle $\mathrm{T}$ antigen mouse via a thrombospondin-1 independent mechanism," Cancer Biology \& Therapy, vol. 5, no. 2, pp. 218-224, 2006.

[156] M. C. Jordan and P. H. Zeplin, "Local inhibition of angiogenesis by halofuginone coated silicone materials," Journal of Materials Science: Materials in Medicine, vol. 23, no. 5, pp. 1203-1210, 2012.

[157] P. A. Assis, L. L. de Figueiredo-Pontes, A. S. G. Lima et al., "Halofuginone inhibits phosphorylation of SMAD-2 reducing angiogenesis and leukemia burden in an acute promyelocytic leukemia mouse model," Journal of Experimental \& Clinical Cancer Research, vol. 34, no. 1, p. 65, 2015.

[158] B. J. Pyun, S. Choi, Y. Lee et al., "Capsiate, a nonpungent capsaicin-like compound, inhibits angiogenesis and vascular permeability via a direct inhibition of Src kinase activity," Cancer Research, vol. 68, no. 1, pp. 227-235, 2008.

[159] S. Chakraborty, A. Adhikary, M. Mazumdar et al., "Capsaicin-induced activation of p53-SMAR1 auto-regulatory loop down-regulates VEGF in non-small cell lung cancer to restrain angiogenesis," PLoS One, vol. 9, no. 6, article e99743, 2014.

[160] F. C. M. Lopes, A. Rocha, A. Pirraco et al., "Anti-angiogenic effects of pterogynidine alkaloid isolated from Alchornea glandulosa," BMC Complementary and Alternative Medicine, vol. 9, no. 1, p. 15, 2009.

[161] J. Tang, Y. Feng, S. Tsao, N. Wang, R. Curtain, and Y. Wang, "Berberine and Coptidis rhizoma as novel antineoplastic agents: a review of traditional use and biomedical investigations," Journal of Ethnopharmacology, vol. 126, no. 1, pp. 517, 2009.

[162] H. Li, G. Tan, X. Jiang et al., "Therapeutic effects of matrine on primary and metastatic breast cancer," The American Journal of Chinese Medicine, vol. 38, no. 6, pp. 1115-1130, 2010.

[163] X. B. Zhao, Y. Qin, Y. L. Niu, and J. Yang, "Matrine inhibits hypoxia/reoxygenation-induced apoptosis of cardiac microvascular endothelial cells in rats via the JAK2/STAT3 signaling pathway," Biomedicine \& Pharmacotherapy, vol. 106, pp. 117-124, 2018.

[164] J. W. Ma, Y. Zhang, R. Li et al., “Tetrandrine suppresses human glioma growth by inhibiting cell survival, proliferation and tumour angiogenesis through attenuating STAT3 phosphorylation," European Journal of Pharmacology, vol. 764, pp. 228-239, 2015.

[165] L. Shi, F. Yang, F. Luo et al., "Evodiamine exerts anti-tumor effects against hepatocellular carcinoma through inhibiting $\beta$-catenin-mediated angiogenesis," Tumor Biology, vol. 37, no. 9, pp. 12791-12803, 2016.

[166] J. P. Eun and G. Y. Koh, "Suppression of angiogenesis by the plant alkaloid, sanguinarine," Biochemical and Biophysical Research Communications, vol. 317, no. 2, pp. 618-624, 2004.

[167] J. K. Min, K. Y. Han, E. C. Kim et al., "Capsaicin inhibits in vitro and in vivo angiogenesis," Cancer Research, vol. 64, no. 2, pp. 644-651, 2004.

[168] Y. Zhang, Q. Jiang, N. Wang, B. Dai, Y. Chen, and L. He, "Effects of taspine on proliferation and apoptosis by regulating caspase- 3 expression and the ratio of $\mathrm{Bax} / \mathrm{Bcl}-2$ in $\mathrm{A} 431$ cells," Phytotherapy Research, vol. 25, no. 3, pp. 357-364, 2011.

[169] T. P. Hamsa and G. Kuttan, "Harmine inhibits tumour specific neo-vessel formation by regulating VEGF, MMP, TIMP and pro-inflammatory mediators both in vivo and in vitro," European Journal of Pharmacology, vol. 649, no. 13, pp. 64-73, 2010.

[170] M. Elkin, R. Reich, A. Nagler et al., "Inhibition of matrix metalloproteinase-2 expression and bladder carcinoma metastasis by halofuginone," Clinical Cancer Research, vol. 5, no. 8, pp. 1982-1988, 1999.

[171] B. Zhao, L. Liu, J. Mao et al., "Sinomenine hydrochloride attenuates the proliferation, migration, invasiveness, angiogenesis and epithelial-mesenchymal transition of clear-cell renal cell carcinoma cells via targeting Smad in vitro," Biomedicine \& Pharmacotherapy, vol. 96, pp. 1036-1044, 2017.

[172] P. Ning, Y. Peng, D. W. Liu, Y. H. Hu, Y. Liu, and D. M. Liu, "Tetrandrine induces microRNA differential expression in human hypertrophic scar fibroblasts in vitro," Genetics and Molecular Research, vol. 15, no. 1, 2016.

[173] M. G. Tonnesen, X. Feng, and R. A. F. Clark, “Angiogenesis in wound healing," Journal of Investigative Dermatology Symposium Proceedings, vol. 5, no. 1, pp. 40-46, 2000.

[174] M. L. Zhu, Y. L. Yin, S. Ping et al., "Berberine promotes ischemia-induced angiogenesis in mice heart via upregulation of microRNA-29b," Clinical and Experimental Hypertension, vol. 39, no. 7, pp. 672-679, 2017.

[175] J. L. Wang, C. Zhang, B. Liu et al., "Function of berberine on porcine in vitro fertilization embryo development and differential expression analysis of microRNAs," Reproduction in Domestic Animals, vol. 54, no. 3, pp. 520-530, 2019.

[176] P.-L. Tsai and T.-H. Tsai, "HPLC determination of berberine in medicinal herbs and a related traditional Chinese medicine," Analytical Letters, vol. 35, no. 15, pp. 24592470, 2002.

[177] J. Yang, F. Gao, Y. Zhang, Y. Liu, and D. Zhang, "Buyang Huanwu decoction (BYHWD) enhances angiogenic effect of mesenchymal stem cell by upregulating VEGF expression after focal cerebral ischemia," Journal of Molecular Neuroscience, vol. 56, no. 4, pp. 898-906, 2015.

[178] C. Huang, H. Liu, X. L. Gong, L. Y. Wu, and B. Wen, "Effect of evodiamine and berberine on the interaction between DNMTs and target microRNAs during malignant transformation of the colon by TGF- $\beta 1$," Oncology Reports, vol. 37, no. 3, pp. 1637-1645, 2017.

[179] P. Caruso, M. R. MacLean, R. Khanin et al., "Dynamic changes in lung microRNA profiles during the development of pulmonary hypertension due to chronic hypoxia and monocrotaline," Arteriosclerosis, Thrombosis, and Vascular Biology, vol. 30, no. 4, pp. 716-723, 2010. 
[180] W. T. Chang, C. H. Hsu, T. L. Huang et al., "MicroRNA-21 is associated with the severity of right ventricular dysfunction in patients with hypoxia-induced pulmonary hypertension," Acta Cardiologica Sinica, vol. 34, no. 6, pp. 511-517, 2018.

[181] L. Q. Li, X. L. Li, L. Wang et al., "Matrine inhibits breast cancer growth via miR-21/PTEN/Akt pathway in MCF-7 cells," Cellular Physiology and Biochemistry, vol. 30, no. 3, pp. 631-641, 2012.

[182] L. Zhao, X. Zhang, and S. Cui, "Matrine inhibits TPC-1 human thyroid cancer cells via the miR-21/PTEN/Akt pathway," Oncology Letters, vol. 16, no. 3, pp. 2965-2970, 2018.

[183] Y. P. Wei, X. H. Wang, G. Liu et al., "Matrine exerts inhibitory effects in melanoma through the regulation of miR19b-3p/PTEN," International Journal of Oncology, vol. 53, no. 2, pp. 791-800, 2018.

[184] A. Tetik Vardarli, Z. Duzgun, C. Erdem, B. T. Kaymaz, Z. Eroglu, and V. B. Cetintas, "Matrine induced G0/G1 arrest and apoptosis in human acute T-cell lymphoblastic leukemia (T-ALL) cells," Bosnian Journal of Basic Medical Sciences, vol. 18, no. 2, pp. 141-149, 2018.

[185] Q. An, C. Han, Y. Zhou et al., "Matrine induces cell cycle arrest and apoptosis with recovery of the expression of miR-126 in the A549 non-small cell lung cancer cell line," Molecular Medicine Reports, vol. 14, no. 5, pp. 4042-4048, 2016.

[186] B. T. Kaymaz, V. B. Çetintaş, C. Aktan, and B. Kosova, "MicroRNA-520a-5p displays a therapeutic effect upon chronic myelogenous leukemia cells by targeting STAT3 and enhances the anticarcinogenic role of capsaicin," Tumor Biology, vol. 35, no. 9, pp. 8733-8742, 2014.

[187] D. S. Zhang, Y. Y. Li, X. J. Chen et al., "BCL2 promotor methylation and miR-15a/16-1 upregulation is associated with sanguinarine-induced apoptotic death in rat HSC-T6 cells," Journal of Pharmacological Sciences, vol. 127, no. 1, pp. 135-144, 2015.

[188] M. Zhou, J. Zeng, X. Wang et al., "MiR-370 sensitizes chronic myeloid leukemia K562 cells to homoharringtonine by targeting Forkhead box M1," Journal of Translational Medicine, vol. 11, no. 1, p. 265, 2013.

[189] L. Song, D. Liu, Y. Zhao et al., "Sinomenine inhibits breast cancer cell invasion and migration by suppressing NF- $\kappa \mathrm{B}$ activation mediated by IL-4/miR-324-5p/CUEDC2 axis," Biochemical and Biophysical Research Communications, vol. 464, no. 3, pp. 705-710, 2015.

[190] T.-P. Fan, J.-C. Yeh, K. W. Leung, P. Y. K. Yue, and R. N. S. Wong, "Angiogenesis: from plants to blood vessels," Trends in Pharmacological Sciences, vol. 27, no. 6, pp. 297-309, 2006.

[191] N. P. Gullett, A. R. M. Ruhul Amin, S. Bayraktar et al., "Cancer prevention with natural compounds," Seminars in Oncology, vol. 37, no. 3, pp. 258-281, 2010.

[192] X. Zhang, L. X. Chen, L. Ouyang, Y. Cheng, and B. Liu, "Plant natural compounds: targeting pathways of autophagy as anticancer therapeutic agents," Cell Proliferation, vol. 45, no. 5, pp. 466-476, 2012. 


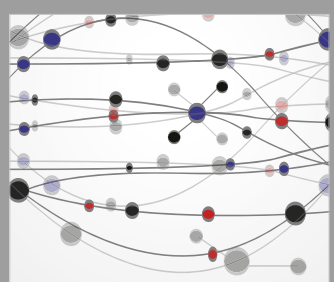

The Scientific World Journal
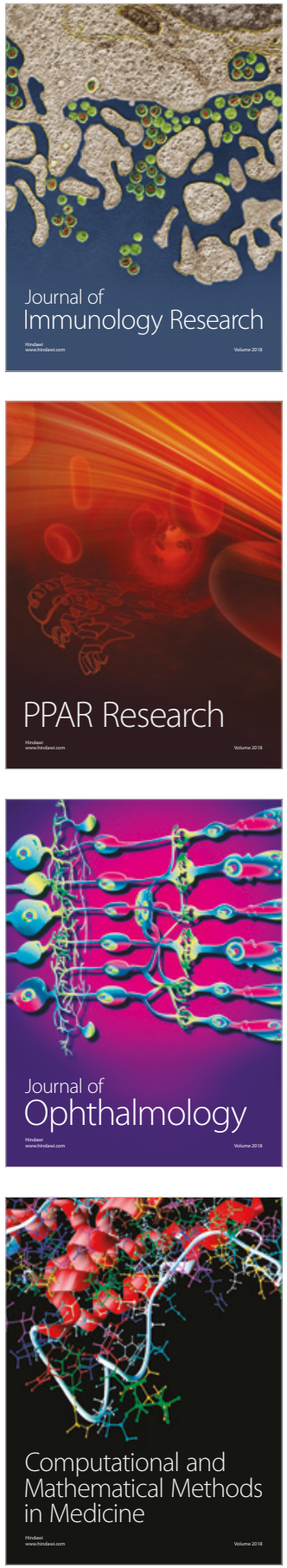

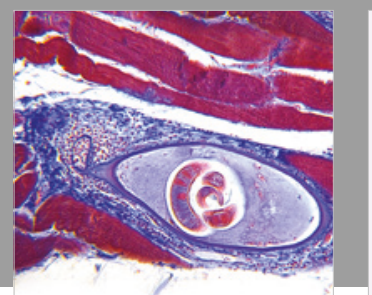

Gastroenterology Research and Practice

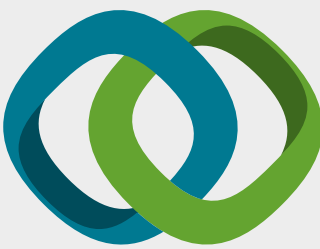

\section{Hindawi}

Submit your manuscripts at

www.hindawi.com
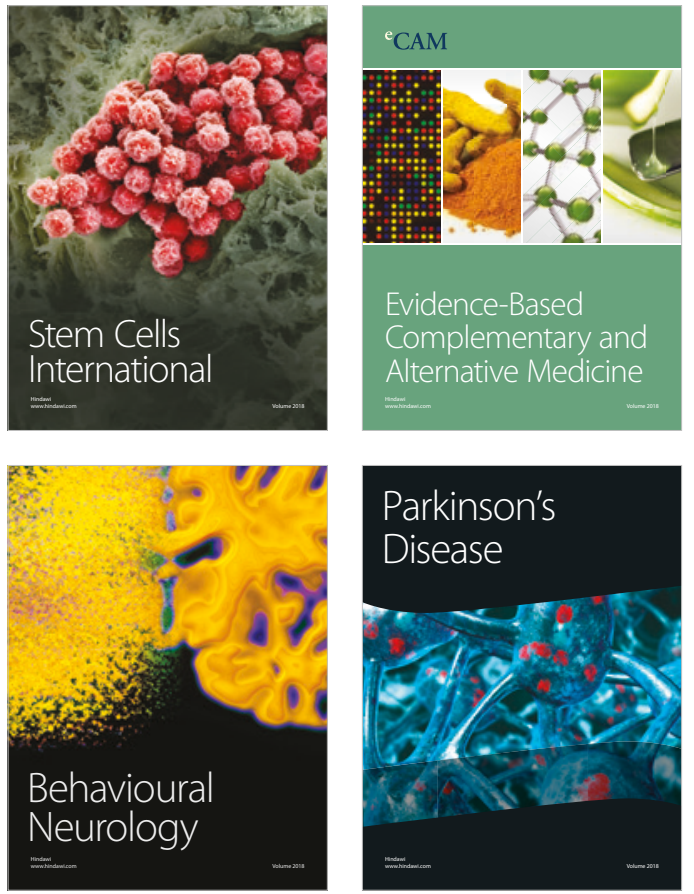

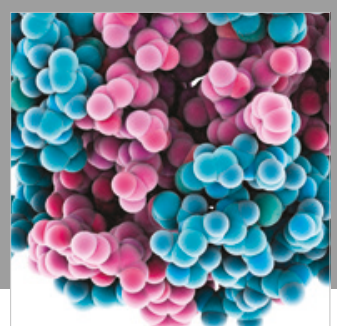

ournal of

Diabetes Research

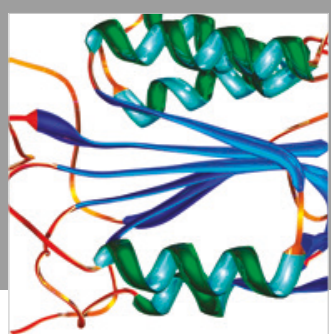

Disease Markers
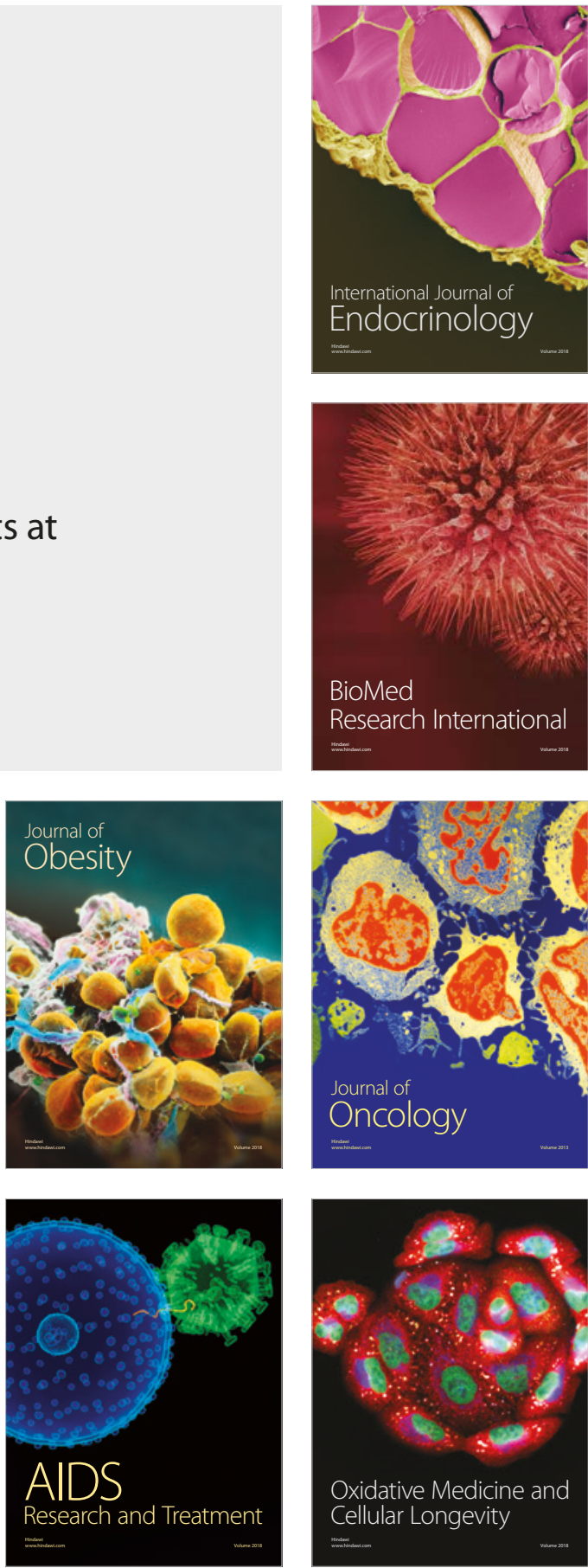